\title{
Global anti-self-dual Yang-Mills fields in split signature and their scattering
}

\author{
L.J.Mason \\ The Mathematical Institute, 24-29 St Giles, Oxford OX1 3LB, England
}

November 15, 2018

\begin{abstract}
This article concerns solutions to the anti-self-dual Yang Mills (ASDYM) equations in split signature that are global on the double cover of the appropriate conformally compactified Minkowski space $\widetilde{\mathbb{M}}=S^{2} \times S^{2}$. Ward's ASDYM twistor construction is adapted to this geometry using a correspondence between points of $\widetilde{\mathbb{M}}$ and holomorphic discs in $\mathbb{C P}^{3}$, twistor space, with boundary on the real slice $\mathbb{R P}^{3}$. Smooth global $\mathrm{U}(n)$ solutions to the ASDYM equations on $\widetilde{\mathbb{M}}$ are shown to be in 1:1 correspondence with pairs consisting of an arbitrary holomorphic vector bundle $E$ over $\mathbb{C P}^{3}$ together with a positive definite hermitian metric $H$ on $\left.E\right|_{\mathbb{R}^{3}}$. There are no topological or other restrictions on the bundle $E$. In ultrahyperbolic signature solutions are generically non-analytic or only finitely differentiable and such solutions arise from a corresponding choice of regularity for $H$. When $E$ is trivial, the twistor data consists of the Hermitian matrix function $H$ on $\mathbb{R P}^{3}$ up to constants and the correspondence provides a nonlinear generalisation of the X-ray transform. In general it provides a higher-dimensional analogue of the (inverse) scattering transform in which $H$ plays the role of the reflection coefficient and $E$ the algebraic data.

Explicit examples are constructed for different choices of the topology of $E$.

A scattering problem for ASDYM fields on affine Minkowski space in split signature is set up and it is shown that sufficiently small data at past null infinity uniquely determines data at future null infinity by taking a family of holonomies associated to the initial data followed by a sequence of two Birkhoff factorizations. The scattering map is simple at the level of the holonomies, but non-trivial at the level of the connection in the non-abelian case.
\end{abstract}

\section{Introduction}

The anti-self-dual Yang-Mills (ASDYM) equations have long been known to be an integrable system, Ward (1977), Belavin \& Zakharov (1978). However, they only admit real solutions in Euclidean (positive definite) signature or split (ultrahyperbolic) signature. The integrability has allowed a substantial study of solutions to the equations in Euclidean signature, see for example Atiyah (1979). Due to the unphysical and peculiar nature of ultrahyperbolic differential equations, the solutions 
in split signature have not been so much studied. Nevertheless, the ASDYM equations in split signature have importance because their symmetry reductions give rise to a wide class of integrable evolution equations, see Ablowitz \& Clarkson (1991) and Mason \& Woodhouse (1996) for surveys. Although the main motivation for this work is mathematical, it is worth noting also that the recent work of Witten (2004) and others has shown that split signature versions of the twistor correspondence provide a useful calculus for twistor string theory with significant applications in perturbative gauge theory. ${ }^{1}$

The purpose of this paper is to adapt the Ward transform to split signature in such a way as to provide a full study of global solutions to the ASDYM equations on the double cover of the conformal compactification of Minkowski space and also of the classical scattering of such fields on affine Minkowski space. The mathematical motivation for the study of the global problem in split signature arises from two sources. The abelian version of the Ward correspondence extends to all massless fields as the Penrose transform between analytic first cohomology classes on regions in twistor space $\mathbb{P} \mathbb{T}=\mathbb{C P}^{3}$ and linear massless fields on corresponding regions in space-time. In split signature, twistor space has a naturally defined real slice $\mathbb{P} \mathbb{T}_{\mathbb{R}}=\mathbb{R P}^{3}$ such that conformally compactified Minkowski space $\mathbb{M}$ is the space of real lines in $\mathbb{R P}^{3}$ (the real Klein correspondence). There is another transform in split signature, the (generalized) X-ray transform: a smooth function $f$ (or section of an appropriate line bundle) on the real slice $\mathbb{P T}_{\mathbb{R}}=\mathbb{R P}^{3} \subset \mathbb{P} \mathbb{T}$ can be integrated along lines in $\mathbb{P T}_{\mathbb{R}}$ to yield a function $\phi$ on ultrahyperbolic Minkowski space. It is a classical result that $\phi$ is a solution to the ultrahyperbolic wave equation and that all such solutions determine a unique $f$ on $\mathbb{R P}^{3}$, John (1938). There is a particular puzzle in that, globally on $\mathbb{C P}^{3}$, the appropriate cohomology group is finite dimensional whereas the X-ray transform shows that there are an infinite dimensional family of solutions. One can nevertheless naively think of the function $f$ as a preferred Cech cocycle, Atiyah (1979). However, the task of finding how this cocycle comes to be preferred and more generally what the precise relationship is between the Penrose and the X-ray transform has led to a substantial literature: see for example Guillemin \& Sternberg (1986), Woodhouse (1992), Mason (1995), Sparling (1998), Bailey, Eastwood, Gover and Mason (1999, 2003), Bailey \& Eastwood (2001) and remarks in 4.1 of this paper. Furthermore, it is this X-ray analogue of the Penrose transform that is predominantly used in Witten (2004). The first motivation then is to find the appropriate non-linear extension of the X-ray transform for ASDYM fields.

The second motivation arises from the theory of integrable systems. For a hyperbolic or parabolic integrable system, the scattering transform usually expresses general data for a solution in terms of a combination of solitonic and radiative or dispersive modes, see for example Faddeev and Takhtajan (1987), Ablowitz and Clarkson (1991). The solitonic modes are usually described by algebreo-geometric data, whereas the radiative/dispersive modes are usually described by smooth functions. The second motivation then is to find the analogous description of the ASDYM equations in split signature. Since many parabolic and hyperbolic integrable systems are symmetry reductions of the ASDYM equations in split signature, this

\footnotetext{
${ }^{1}$ however, the use of the 'wrong signature' has also perhaps led to some of the technical problems of twistor-string theory.
} 
would give some general insight as to how such constructions arise from their twistor descriptions.

The main theorem is a correspondence for solutions to the anti-self-dual YangMills (ASDYM) equations with compact gauge group $G$ on the double cover of the conformal compactification of ultrahyperbolic Minkowski space $\widetilde{\mathbb{M}}=S^{2} \times S^{2}$. The correspondence is with certain data on complex projective three space $\mathbb{C P}^{3}$, the twistor space which we denote by $\mathbb{P} \mathbb{T}$, and its real slice $\mathbb{R} \mathbb{P}^{3}$ denoted $\mathbb{P} \mathbb{T}_{\mathbb{R}}$. We have

Theorem 1 Gauge equivalence classes of $C^{\infty}$ solutions to the ASDYM equations on $\widetilde{\mathbb{M}}$ are in 1-1 correspondence with principal $G_{\mathbb{C}}$ bundles $P \rightarrow \mathbb{P T}$ together with a $C^{\infty}$ reduction of the structure group to $G$ over $\mathbb{P T}_{\mathbb{R}}$ where $G_{\mathbb{C}}$ is the complexification of $G$. The reduction of the structure group over $\mathbb{P T}_{\mathbb{R}}$ can be expressed as a section $H: \mathbb{P T}_{\mathbb{R}} \rightarrow P / G$ of the $G_{\mathbb{C}} / G$ bundle $P / G$.

In the $\mathrm{U}(n)$ case, $P$ is the principal bundle associated to a holomorphic vector bundle $E$, and $H$ defines a hermitian metric on the fibres of $E$ restricted to $\mathbb{P T}_{\mathbb{R}}$ (when $G=\mathrm{U}(n), G_{\mathbb{C}} / G$ is the space $\mathrm{Herm}_{n}$ of $n \times n$ positive definite hermitian matrices).

The techniques extend to the non smooth case: $C^{k, \alpha}$ solutions to the ASDYM equations arise when $H$ is in $C^{k+1, \alpha^{\prime}}$ for $\alpha^{\prime}>\alpha$. However, the techniques of the proof lose too many derivatives in the forward direction to give a definitive theorem whereas to obtain a definitive result one would need to gain at least one.

The theorem gives the space $\mathcal{S}_{G}$ of gauge equivalence classes of solutions to the ASDYM equations on $\widetilde{\mathbb{M}}$ the structure of a fibre bundle $\pi: \mathcal{S}_{G} \rightarrow \mathcal{H}_{G_{\mathbb{C}}}$ where $\mathcal{H}_{G_{\mathbb{C}}}$ is the space of holomorphic $G_{\mathbb{C}}$ bundles over $\mathbb{P} \mathbb{T}$ and the fibre $\pi^{-1}(P)$ at $P \in \mathcal{H}_{G_{\mathbb{C}}}$ is the space of sections $H: \mathbb{P T}_{\mathbb{R}} \rightarrow P / G$. The space $\mathcal{H}_{G_{\mathbb{C}}}$ has many components labelled by the possible topological types of $P$. The different components are not, however, smooth manifolds as arbitrary holomorphic bundles are allowed, including unstable ones. Ignoring these subtleties, the individual connected components of $\mathcal{H}_{G_{\mathbb{C}}}$ are finite dimensional. However, at a given $P \in \mathcal{H}_{G_{\mathbb{C}}}$, the fibre $\pi^{-1}(P)=\Gamma\left(\mathbb{P T}_{\mathbb{R}}, P / G\right)$ is infinite dimensional being a twisted analogue of smooth maps from $\mathbb{R P}^{3}$ to $G_{\mathbb{C}} / G$ modulo at worst a finite-dimensional equivalence under the global automorphisms $\operatorname{Aut}(P)$ of $P$. When $P$ or $E$ is trivial $\operatorname{Aut}(P)=G_{\mathbb{C}}$, the corresponding component of $\mathcal{H}_{G_{\mathbb{C}}}$ is a point and so the fibre is the quotient of the space of smooth maps $\left\{\right.$ Maps: $\mathbb{P T}_{\mathbb{R}} \rightarrow G_{\mathbb{C}} / G$ \} divided on the left by $G_{\mathbb{C}}$. Thus, for trivial $P$, the theorem gives a direct nonlinear analogue of the X-ray transform. It also gives the appropriate generalisation of the scattering transform for ASDYM fields with arbitrary $P$ as then the space $\mathcal{H}_{G_{\mathbb{C}}}$ is the appropriate algebreo-geometric generalisation of the solitonic data, and $H$ is the appropriate generalisation of the reflection coefficient describing the radiative/dispersive modes of the field.

The motivation for the study of scattering arises from a number of areas. It has long been suggested that scattering for integrable systems in dimensions greater than $1+1$ should be trivial partly as a consequence of Huygens principle, and as a consequence of calculations in perturbation theory. This is very much not the case for Ward's integrable chiral model in $2+1$ where there is right-angle scattering of lumps and this is a symmetry reduction of the ASDYM equations in split signature. Scattering for the ASDYM equations is trivial perturbatively in Lorentz signature, but in the complex, and in particular in split signature, the relevant amplitudes do 
not vanish. Indeed it follows from the theorem above that in the case of trivial $P$ but non-trivial $H$, if one makes a choice of past and future infinity, although the data on one of future or past infinity determines the whole field, the field obtained at future infinity is different from that at past infinity and this is here developed into a study of the scattering problem. The original motivation of the author was to make some contact with the perturbative calculations of scattering in Witten (2004) but the ASD sector is effectively suppressed there (as appropriate for scattering in Lorentz signature).

The principal tool is a generalisation of the Ward construction adapted to split signature. The standard Ward construction uses a correspondence between points in space-time and Riemann spheres (complex projective lines) in twistor space and encodes the original ASDYM field into a holomorphic vector bundle over (a region in) $\mathbb{P T}$. This generalisation uses a correspondence between points of space-time and certain holomorphic discs in twistor space with boundary on the real slice $\mathbb{P} \mathbb{T}_{\mathbb{R}}$.

The paper is constructed as follows. In the basic geometry of compactified ultrahyperbolic Minkowski space $\mathbb{M}$ and its double cover $\widetilde{\mathbb{M}}$ is set up together with its correspondence with complex twistor space $\mathbb{P T}$ and its real slice $\mathbb{P T}_{\mathbb{R}}$. In particular points of $\widetilde{\mathbb{M}}$ correspond to oriented lines in $\mathbb{P T}_{\mathbb{R}}=\mathbb{R P}^{3}$ that bound holomorphic discs in $\mathbb{P T}$. In $\$ 3$ the main result Theorem 1 is proved and some of its consequences are explored. In $\$$ we discuss various examples corresponding to different choices of the topology of $E$ etc., and those solutions that correspond to the pullback of ASDYM fields that are pulled back from $\mathbb{M}=S^{2} \times S^{2} / \mathbb{Z}_{2}$. Only the abelian examples are given explicitly in this section - the non-abelian examples are discussed and shown to exist here, but are only given in full detail in an appendix as a Kahler formalism is required to express the solutions straighforwardly and this is only presented in the appendix.

In $\$ 5$ the construction is applied to the task of calculating the scattering of characteristic data from past null infinity, $\mathscr{I}^{-}$, to future null infinity, $\mathscr{I}^{+}$when the data is small. ${ }^{2}$ The scattering can be expressed simply in terms of certain holonomies associated to the connection that is presented as initial data. The holonomies $h$ of the connection around a 3 parameter family of loops (two oriented loops for each point in real twistor space) encodes the original data when both are small by a theorem of Novikov (2002). This $h$ is then related to the twistor data as derived in 93 by a Birkhoff factorization on a family of lines in the twistor space. Generically, the scattering will be nontrivial in the non-abelian case, being $h \rightarrow h^{-1}$. In the abelian case it reduces simply to a sign reversal of the connection, but will be nontrivial in the non-abelian case, requiring a sequence of two Birkhoff factorizations to calculate the effect on the potentials. It is worth noting that in perturbation theory, the amplitudes for the self-dual sector formally vanish in Minkowski signature, but are non-trivial in the complex and in particular in split signature.

In 6 some further avenues are discussed. In the first apendix a technical lemma required in the proof of theorem 2 is proved. In the second a formalism for the twistor correspondence for $\widetilde{\mathbb{M}}$ is developed adapted to a choice of a complex structure on $\widetilde{\mathbb{M}}$ given by an identification with $\mathbb{C P}^{1} \times \mathbb{C P}^{1}$. This is then used to give an explicit description of the ADHM construction adapted to split signature to give global

${ }^{2}$ The division of null infinity $\mathscr{I}$ into future and past is not canonical in split signature, but we will see that the scattering problem can nevertheless be made sense of. 
solutions with second Chern class 2, and to work through an example of the Ward ansatze.

Finally we give some references to earlier and related work on these issues. Lerner (1992) introduced a similar such $H$ to describe ASD Yang-Mills fields in split signature but did not fix the global behaviour and so does not incorporate the bundle $E$ or the gauge fixing that arises when $E$ is trivial. The methods used here are a development of those presented in Mason (1995) and $\S 10.5$ of Mason \& Woodhouse (1996) (which emphasized a non-Hausdorff twistor space construction). A key improvement in this paper is the use of holomorphic discs with boundary on $\mathbb{R P}^{3}$ and this arises from analogous work in lower dimension with Claude LeBrun on Zoll projective structures in dimension 2, LeBrun and Mason (2002). Similar methods apply to the split signature version of the nonlinear graviton construction, Penrose (1976), for anti-self-dual conformal structures on $S^{2} \times S^{2}$ and this is treated in a separate joint paper with Claude LeBrun, LeBrun \& Mason (2005).

Acknowledgements: I would like to thank Claude LeBrun for a number of important contributions to this work. Thanks are also due to Toby Bailey, Maciej Dunajski, Mike Eastwood, Gavin Kelly, Elmer Rees, George Sparling, Nick Woodhouse and the anonymous referee. I would also like to thank the Department of Mathematical Sciences at the University of Edinburgh for hospitality for some of the time while this work was being written up.

\section{Twistors in split signature}

\subsection{Conformally compactified Minkowski space}

We denote signature $(p, q)$ Minkowski space by $\mathbb{R}^{p, q}$ which is $\mathbb{R}^{p+q}$ with a flat metric of signature $(p, q)$. The standard conformal compactification $\mathbb{M}^{p, q}$ of $\mathbb{R}^{p, q}$ is obtained by adding a 'lightcone at infinity', denoted $\mathscr{I}$ and has a standard representation as the projectivisation of the lightcone of the origin of $\mathbb{R}^{p+1, q+1}$. We will only be concerned with $\mathbb{M}^{2,2}$ which we will denote by $\mathbb{M}$ : it is a projective quadric of signature $(3,3)$ in $\mathbb{R} \mathbb{P}^{5}$. It is easily seen to have topology $S^{2} \times S^{2} / \mathbb{Z}_{2}$ by choosing coordinates $(\mathbf{x}, \mathbf{y})$ on $\mathbb{R}^{6}, \mathbf{x}, \mathbf{y} \in \mathbb{R}^{3}$, such that the quadratic form is $Q=\mathbf{x} \cdot \mathbf{x}-\mathbf{y} \cdot \mathbf{y}$ and $\cdot$ denotes the standard positive definite inner product on $\mathbb{R}^{3}$. Then the light cone of the origin is given by $Q=0$ which gives $\mathbf{x} \cdot \mathbf{x}=\mathbf{y} \cdot \mathbf{y}$ and we can normalize $\mathbf{x} \cdot \mathbf{x}=\mathbf{y} \cdot \mathbf{y}=1$. Clearly this gives $S^{2} \times S^{2}$ in $\mathbb{R}^{6}$, and the projection to $Q=0$ in $\mathbb{R} \mathbb{P}^{5}$ is the quotient by the joint antipodal map $\tilde{\sigma}$ on each $S^{2}, \tilde{\sigma}:(\mathbf{x}, \mathbf{y}) \rightarrow(-\mathbf{x},-\mathbf{y})$. The conformal structure on $\mathbb{M}$ is determined by requiring that the lines in $\mathbb{R} \mathbb{P}^{5}$ that lie on $Q=0$ are the null geodesics of $\mathbb{M}$.

We will also be interested in the double cover, $\widetilde{\mathbb{M}}=S^{2} \times S^{2}$ of $\mathbb{M}$ with covering map $\sigma: \widetilde{\mathbb{M}} \rightarrow \mathbb{M}$. Concretely we can parametrize $\widetilde{\mathbb{M}}$ by complex stereographic coordinates $w_{1}$ and $w_{2} \in \mathbb{C}$ so that

$$
\mathbf{x}=\frac{\left(w_{1}+\bar{w}_{1}, i \bar{w}_{1}-i w_{1}, 1-\left|w_{1}\right|^{2}\right)}{\left(1+\left|w_{1}\right|^{2}\right)} \quad \text { and } \quad \mathbf{y}=\frac{\left(w_{2}+\bar{w}_{2}, i \bar{w}_{2}-i w_{2}, 1-\left|w_{2}\right|^{2}\right)}{\left(1+\left|w_{2}\right|^{2}\right)}
$$

and

$$
\mathrm{d} s^{2}=\frac{4 \mathrm{~d} w_{1} \mathrm{~d} \bar{w}_{1}}{\left(1+\left|w_{1}\right|^{2}\right)^{2}}-\frac{4 \mathrm{~d} w_{2} \mathrm{~d} \bar{w}_{2}}{\left(1+\left|w_{2}\right|^{2}\right)^{2}}
$$


If we take the volume form to be $\mathrm{e}^{3}$

$$
\mathrm{d} \operatorname{Vol}=\frac{\mathrm{d} w_{1} \wedge \mathrm{d} \bar{w}_{1} \wedge \mathrm{d} w_{2} \wedge \mathrm{d} \bar{w}_{2}}{\left(1+\left|w_{1}\right|^{2}\right)^{2}\left(1+\left|w_{1}\right|^{2}\right)^{2}}
$$

then the self-dual 2-forms are spanned by

$$
\mathrm{d} w_{1} \wedge \mathrm{d} w_{2}, \quad \mathrm{~d} \bar{w}_{1} \wedge \mathrm{d} \bar{w}_{2}, \quad \text { and } \quad \frac{4 \mathrm{~d} w_{1} \wedge \mathrm{d} \bar{w}_{1}}{\left(1+\left|w_{1}\right|^{2}\right)^{2}}-\frac{4 \mathrm{~d} w_{2} \wedge \mathrm{d} \bar{w}_{2}}{\left(1+\left|w_{2}\right|^{2}\right)^{2}}
$$

and the anti-self-dual forms by

$$
\mathrm{d} w_{1} \wedge \mathrm{d} \bar{w}_{2}, \quad \mathrm{~d} \bar{w}_{1} \wedge \mathrm{d} w_{2}, \quad \text { and } \quad \frac{4 \mathrm{~d} w_{1} \wedge \mathrm{d} \bar{w}_{1}}{\left(1+\left|w_{1}\right|^{2}\right)^{2}}+\frac{4 \mathrm{~d} w_{2} \wedge \mathrm{d} \bar{w}_{2}}{\left(1+\left|w_{2}\right|^{2}\right)^{2}}
$$

\section{$2.2 \quad$ Twistors}

The twistor correspondence is the real Klein correspondence in which each point $p \in \mathbb{M}$ corresponds to a line $L_{p}$ in the real twistor space $\mathbb{P T}_{\mathbb{R}}=\mathbb{R P}^{3}$ (here $\mathbb{T}_{\mathbb{R}}$ denotes real non-projective twistor space, $\mathbb{R}^{4}$ and $\mathbb{T}$ its complexification, $\mathbb{C}^{4}$ ). The correspondence follows by representing a line in $\mathbb{P T}_{\mathbb{R}}$ by a 2 -plane through the origin in $\mathbb{T}_{\mathbb{R}}$ and then parametrizing such 2-planes by simple bivectors $X \in \wedge^{2} \mathbb{T}_{\mathbb{R}}=\mathbb{R}^{6}$ up to scale. The simplicity condition is $X \wedge X=0$ which defines the quadric $\mathbb{M} \subset \mathbb{P}\left(\wedge^{2} \mathbb{T}_{\mathbb{R}}\right)=\mathbb{R} \mathbb{P}^{5}$. Under this correspondence, points of $\widetilde{\mathbb{M}}$ correspond to oriented lines in $\mathbb{P} \mathbb{T}_{\mathbb{R}}$.

Twistor theory makes essential use of the complexification $\mathbb{P T}=\mathbb{C P}^{3}$ of $\mathbb{P T}_{\mathbb{R}}$. Each point $x \in \widetilde{\mathbb{M}}$ corresponds to a holomorphic closed disc $D_{x} \subset \mathbb{P T}$ lying in the complexification $\mathbb{C} L_{\sigma(x)}$ of $L_{\sigma(x)}$ (denoting the image of $x$ in $\mathbb{M}$ by $\sigma(x)$ ) such that $\partial D_{x}=L_{\sigma(x)}$ and so that the induced complex structure on $D_{x}$ induces the appropriate orientation on the boundary corresponding to $x$.

A real twistor $Z \in \mathbb{P T}_{\mathbb{R}}$ (resp. dual twistor $W \in \mathbb{P T}_{\mathbb{R}}^{*}$ ) corresponds in $\mathbb{M}$ to a totally null self-dual (resp. anti-self-dual) two-plane, referred to as an $\alpha$-plane (resp. $\beta$-plane) corresponding to the lines in $\mathbb{P} \mathbb{T}_{\mathbb{R}}$ through $Z$ (resp. lines lying in the plane corresponding to $W){ }^{4}$

In order to expedite the correspondence, we introduce the (six-dimensional) correspondence space $\mathscr{F}=\left\{(x, Z) \in \widetilde{\mathbb{M}} \times \mathbb{P} \mathbb{T} \mid Z \in D_{x}\right\}$ which naturally fibres over both $\mathbb{P} \mathbb{T}$ and $\widetilde{\mathbb{M}}$.

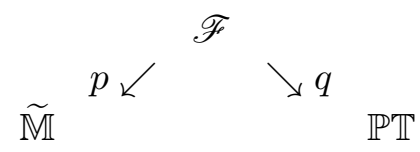

The fibre of $p: \mathscr{F} \rightarrow \widetilde{\mathbb{M}}$ at $x \in \widetilde{\mathbb{M}}$ is the corresponding disc $D_{x} \subset \mathbb{P} \mathbb{T}$.

The real correspondence space $\mathscr{F}_{\mathbb{R}}=\left\{(x, Z) \in \widetilde{\mathbb{M}} \times \mathbb{P T}_{\mathbb{R}} \mid Z \in \partial D_{x}\right\}$ is the 5dimensional boundary of $\mathscr{F}$ and fibres over $\mathbb{P T}_{\mathbb{R}}$ with fibres consisting of lifts of $\alpha$-planes with topology $S^{2}$. For the fibre of $q: \mathscr{F} \rightarrow \mathbb{P T}$ at $Z \notin \mathbb{P} \mathbb{T}_{\mathbb{R}}$ we have

\footnotetext{
${ }^{3}$ In LeBrun \& Mason 2005 the opposite and more natural sign is taken for the volume form; the conventions used here are consistent with those of Mason \& Woodhouse (1996).

${ }^{4}$ In $\widetilde{\mathbb{M}}$ the general $\alpha$-plane, with our conventions, is the graph of an orientation reversing isometry from one $S^{2}$ factor to the other, and the general $\beta$-plane is the graph of an orientation preserving isometry from one $S^{2}$ factor to the other. The above representation identifies real twistor space $\mathbb{P T}_{\mathbb{R}}$ with $\mathrm{SO}(3)=\mathrm{PSU}(2)$. Complex non-projective twistor space $\mathbb{T}$ can be represented as the space of complex non-vanishing $2 \times 2$ matrices with real slice $\mathbb{T}_{\mathbb{R}}$ being given by those that are unitary up to a real scale. This description is taken further in the appendix.
} 
Lemma $2.1 q: \mathscr{F}-\mathscr{F}_{\mathbb{R}} \rightarrow \mathbb{P} T-\mathbb{P T}_{\mathbb{R}}$ is $1: 1$ and onto.

Proof: This follows from the fact that, given $Z \in \mathbb{P T}-\mathbb{P} \mathbb{T}_{\mathbb{R}}$, there is a unique real line $L \subset \mathbb{P T}_{\mathbb{R}}$ whose complexification $\mathbb{C} L$ contains $Z$ since $\mathbb{C} L$ must also contain $\bar{Z}$ and therefore be the line joining $Z$ to $\bar{Z}$. Furthermore, $Z$ determines the disc $D \subset \mathbb{C} L$ containing $Z$ with boundary $L$ and so this $D$ corresponds to an $x \in \widetilde{\mathbb{M}}$. Thus $Z$ determines $x$ together with $Z \in D_{x}$.

Corollary 2.1 $\mathscr{F}-\mathscr{F}_{\mathbb{R}}$ has a natural complex structure from its identification with $\mathbb{P T}-\mathbb{P}_{\mathbb{R}}$

Remark: Analogous to the Atiyah-Hitchin-Singer definition of twistor space, we note that $\mathscr{F}-\mathscr{F}_{\mathbb{R}}$ has a natural interpretation as the bundle of metric compatible complex structures on $\widetilde{\mathbb{M}}$ and its complex structure can be defined as in Atiyah, Hitchin and Singer (1978). However, on $\mathscr{F}_{\mathbb{R}}$ the distribution defining the complex structure has a real part and so the description breaks down.

\subsection{Coordinates on an affine chart}

If we we send the light cone $\mathscr{I}$ of a point $i$ to $\infty$ we are left with an affine chart $\mathbb{R}^{2,2} \subset \mathbb{M}$ on which we can introduce standard Penrose notation, Penrose \& Rindler (1984 \& 1986) adapted to split signature. Here we take the corresponding points in $\widetilde{\mathbb{M}}$ to be $i^{-}$given by $\left(w_{1}, w_{2}\right)=(0,0)$ or its antipode $i^{+}=(\infty, \infty)$ and $\mathscr{I}$ is the hypersurface $\left|w_{1}\right|=\left|w_{2}\right|$. Taking out $\mathscr{I}$ divides $\widetilde{\mathbb{M}}$ into two copies $\mathbb{M}^{ \pm}=$ $\left\{ \pm\left(\left|w_{1}\right|-\left|w_{2}\right|\right)>0\right\}$ of $\mathbb{R}^{4}$, one of which, say $\mathbb{M}^{+}$, can be taken to be 'physical' space-time. We use affine coordinates on $\mathbb{M}^{+}$which can be expressed in terms of $(\mathbf{x}, \mathbf{y})=\left(x_{1}, x_{2}, x_{3}, y_{1}, y_{2}, y_{3}\right)$ as

$$
x^{A A^{\prime}}=\frac{1}{\sqrt{2}\left(x_{3}-y_{3}\right)}\left(\begin{array}{cc}
x_{1}-y_{1} & x_{2}+y_{2} \\
-x_{2}+y_{2} & x_{1}+y_{1}
\end{array}\right), \quad A=0,1, A^{\prime}=0^{\prime}, 1^{\prime} .
$$

Here spinor indices $A, A^{\prime}$ are raised and lowered with the skew-symmetric spinors $\varepsilon_{A B}=-\varepsilon_{B A}$ and $\varepsilon_{A^{\prime} B^{\prime}}, \varepsilon_{01}=\varepsilon_{0^{\prime} 1^{\prime}}=1$ and so transform under $\operatorname{SL}(2, \mathbb{R})$. The conformal structure can be represented by the metric

$$
\mathrm{d} s^{2}=\mathrm{d} x^{A A^{\prime}} \mathrm{d} x^{B B^{\prime}} \varepsilon_{A B} \varepsilon_{A^{\prime} B^{\prime}} .
$$

We can then introduce complex homogenous coordinates $\left(\omega^{A}, \pi_{A^{\prime}}\right)$ on $\mathbb{P T}$ (which, for real values restrict to real homogeneous coordinates on $\left.\mathbb{P T}_{\mathbb{R}}\right)$. Then the correspondence is given by the incidence relation

$$
\omega^{A}=x^{A A^{\prime}} \pi_{A^{\prime}}
$$

which can be read either as an equation defining the $\alpha$-plane in $\mathbb{M}^{+}$for fixed $\left(\omega^{A}, \pi_{A^{\prime}}\right)$ or as an equation defining a projective line in $\mathbb{P} \mathbb{T}_{\mathbb{R}}$ for fixed $x^{A A^{\prime}}$.

The holomorphic discs in $\mathbb{P T}$ with boundary on $\mathbb{P T}_{\mathbb{R}}$ that correspond to points of $\mathbb{M}^{ \pm}$can be parametrized by complex homogeneous coordinates $\pi_{A^{\prime}}$ subject to $\pm i \pi_{A^{\prime}} \bar{\pi}^{A^{\prime}} \geq 0$ and $\omega^{A}$ given by ([6). In the + case, the homogeneous coordinates are related to the standard disc coordinate $z$ with $|z| \leq 1$ by $z=\left(\pi_{0^{\prime}}+i \pi_{1^{\prime}}\right) /\left(\pi_{0^{\prime}}-i \pi_{1^{\prime}}\right)$.

The spin bundle $\mathbb{S}$ has coordinates $\left(x^{A A^{\prime}}, \pi_{A^{\prime}}\right)$ and the restriction of $\mathscr{F}$ to $\mathbb{M}^{+}$can be identified with the subset of the projective spin bundle $\mathbb{P S}$ on which $i \pi_{A^{\prime}} \bar{\pi}^{A^{\prime}} \geq 0$ 
with equality on $\mathscr{F}_{\mathbb{R}}$. The fact that the map from $\mathscr{F}-\mathscr{F}_{\mathbb{R}}$ is $1-1$ can be expressed as the fact that given $\left(\omega^{A}, \pi_{A^{\prime}}\right)$ with $i \pi_{A^{\prime}} \bar{\pi}^{A^{\prime}}>0$ equation (6) has the unique real solution

$$
x^{A A^{\prime}}=\frac{\omega^{A} \bar{\pi}^{A^{\prime}}+\bar{\omega}^{A} \pi^{A^{\prime}}}{i \pi_{B^{\prime}} \bar{\pi}^{B^{\prime}}} .
$$

On $\mathbb{S}$ we define the twistor distribution $\mathcal{D}=\left\{\partial / \partial \bar{\pi}_{A^{\prime}}, \pi^{A^{\prime}} \partial_{A A^{\prime}}\right\}$ and this descends also to $\mathbb{P S}$. This is conformally invariant. On $\mathscr{F}-\mathscr{F}_{\mathbb{R}} \operatorname{dim}\{\mathcal{D} \cap \overline{\mathcal{D}}\}=0$, the projection $q$ is $1: 1$ and and $\mathcal{D}$ descends to give $T^{0,1}$ for the standard complex structure on $\mathbb{P T}-\mathbb{P T}_{\mathbb{R}}$. The d-bar operator can be written

$$
\bar{\partial}=\mathrm{d} \bar{\pi}_{A^{\prime}} \frac{\partial}{\partial \bar{\pi}_{A^{\prime}}}+\frac{1}{\pi^{A^{\prime}} \bar{\pi}_{A^{\prime}}} \mathrm{d} x^{A A^{\prime}} \bar{\pi}_{A^{\prime}} \pi^{B^{\prime}} \partial_{A B^{\prime}}
$$

On $\mathscr{F}_{\mathbb{R}}, \operatorname{dim}\{\mathcal{D} \cap \overline{\mathcal{D}}\}=2$ and $\mathcal{D} \cap \overline{\mathcal{D}}=\left\{\pi^{A^{\prime}} \partial_{A A^{\prime}}\right\}$ is then tangent to the 2-dimensional fibres of the projection $q: \mathscr{F}_{\mathbb{R}} \rightarrow \mathbb{P} \mathbb{T}_{\mathbb{R}}$.

\subsection{The ASDYM equations and Lax pair}

The ASDYM equations are equations on a connection $D$ on a bundle $E^{\prime} \rightarrow \widetilde{\mathbb{M}}$. In a given local trivialisation $D=\mathrm{d}+A$ where $A=A_{A A^{\prime}} \mathrm{d} x^{A A^{\prime}} \in \Omega^{1}(\widetilde{\mathbb{M}}) \otimes \mathrm{u}(n)$ for a $\mathrm{U}(n)$ connection. The curvature is $F=D^{2}=\mathrm{d} A+A \wedge A$. The ASDYM equations are the condition that the curvature satisfies the anti-self-duality condition $F^{*}=-F$ where $*$ is the Hodge dual, $F_{a b}^{*}=\frac{1}{2} \varepsilon_{a b}{ }^{c d} F_{c d}$. The curvature naturally decomposes into its self-dual and anti-self-dual parts when expressed in spinors

$$
F_{A A^{\prime} B B^{\prime}}=\varepsilon_{A B} \phi_{A^{\prime} B^{\prime}}+\varepsilon_{A^{\prime} B^{\prime}} \phi_{A B}
$$

where $\phi_{A^{\prime} B^{\prime}}=\partial_{\left(A^{\prime}\right.}^{A} A_{\left.B^{\prime}\right) A}+A_{\left(A^{\prime}\right.}^{A} A_{\left.B^{\prime}\right) A}$ (resp. $\left.\phi_{A B} \partial_{(A}^{A^{\prime}} A_{B) A^{\prime}}+A_{\left(A^{\prime}\right.}^{A^{\prime}} A_{B) A^{\prime}}\right)$ is the selfdual (resp. anti-self-dual) part of the curvature. A connection is ASD iff $\phi_{A^{\prime} B^{\prime}}=0$.

A Lax pair for the ASDYM equations on $D$ is given by $\pi^{A^{\prime}} D_{A A^{\prime}}$ in the sense that $\left[\pi^{A^{\prime}} D_{A A^{\prime}}, \pi^{B^{\prime}} D_{B B^{\prime}}\right]=0$ iff the ASDYM equations hold (Ward 1977).

\section{Global ASDYM fields in split signature}

\subsection{The generalised Ward correspondence}

Our main theorem for $\mathrm{U}(n)$ ASDYM fields on $\widetilde{\mathbb{M}}$ is as follows

Theorem 2 There is a 1-1 correspondence between smooth $\mathrm{U}(n)$ ASDYM fields on $\widetilde{\mathbb{M}}$ and pairs $(E, H)$ where $E$ is a rank $n$ holomorphic vector bundles on twistor space $\mathbb{P T}$ and $H$ is a smooth positive definite hermitian metric on the fibres of $\left.E\right|_{\mathbb{R P}^{3}}$.

If there exists an antilinear conjugation $\tilde{\sigma}_{E}: E \rightarrow \bar{E}^{*}$ covering the standard complex conjugation $\tilde{\sigma}: \mathbb{P} \mathbb{T} \rightarrow \mathbb{P T}$ that fixes $\mathbb{P} \mathbb{T}_{\mathbb{R}}$, such that $H$ is induced by $\tilde{\sigma}_{E}$ by $H(v, v)=\left(\tilde{\sigma}_{E} v\right)(\bar{v})$ then the ASDYM field on $\widetilde{\mathbb{M}}$ is one that is pulled back from $\mathbb{M}$.

A technical lemma required in the proof of this theorem is relegated to the first appendix.

Proof (forward direction): We are given an ASDYM connection $D=\mathrm{d}+A$ on the bundle $E^{\prime} \rightarrow \widetilde{\mathbb{M}}$ where $A$ is a 1 -form on $\mathbb{M}$ with values in the Lie algebra of 
$\mathrm{U}(n)$. In order to produce the pair $(E, H)$ where $E$ is a holomorphic vector bundle over $\mathbb{P} \mathbb{T}$ and $H$ is a Hermitian metric on $E \mid \mathbb{P} \mathbb{T}_{\mathbb{R}}$, define first $E \rightarrow \mathbb{P}-\mathbb{P T}_{\mathbb{R}}$ by $E=\left(q^{-1}\right)^{*} p^{*} E^{\prime}$ where $p$ and $q$ are the projections of the double fibration (4). The connection $D$ lifts to give a connection on $p^{*} E^{\prime} \rightarrow \mathscr{F}$, and, away from $\partial \mathscr{F}$, the map $q$ is $1: 1$ and hence this determines a connection on $E=\left(q^{-1}\right)^{*} p^{*} E^{\prime}$ over $\mathbb{P T}-\mathbb{P T}_{\mathbb{R}}$. The connection determines a $\bar{\partial}$-operator on $E$ which can be represented in the affine coordinates above as

$$
\bar{\partial}_{E}=\mathrm{d} \bar{\pi}_{A^{\prime}} \frac{\partial}{\partial \bar{\pi}_{A^{\prime}}}+\frac{1}{\pi^{A^{\prime}} \bar{\pi}_{A^{\prime}}} \mathrm{d} x^{A A^{\prime}} \bar{\pi}_{A^{\prime}} \pi^{B^{\prime}} D_{A B^{\prime}} .
$$

It is a standard calculation that $\bar{\partial}_{E}^{2}=0$ as a consequence of the ASDYM equations based on 2.4 and so $\left(E, \bar{\partial}_{E}\right)$ is a holomorphic vector bundle over $\left.E\right|_{\mathbb{P} \mathbb{T}-\mathbb{P} \mathbb{T}_{\mathbb{R}}}$.

We now define $E \rightarrow \mathbb{P T}_{\mathbb{R}}$ to be the bundle whose fibre at $Z \in \mathbb{P T}_{\mathbb{R}}$ is the space of covariantly constant sections over the corresponding $\alpha$-plane in $\widetilde{\mathbb{M}}$ (the $\alpha$-planes in $\widetilde{\mathbb{M}}$ are simply connected, being $S^{2}$ s, and the anti-self-duality condition implies that the curvature vanishes on each real $\alpha$-plane so the space of covariantly constant sections is well defined). Clearly, $\left.E\right|_{\mathbb{P}_{\mathbb{R}}}$ carries a hermitian metric $H$.

The definitions of $E$ over $\mathbb{P T}-\mathbb{P T}_{R}$ and over $\mathbb{P T}_{\mathbb{R}}$ are quite different but we have

Lemma 3.1 The given definition of $E \rightarrow \mathbb{P T}_{\mathbb{R}}$ is a smooth extension of $E \rightarrow$ $\mathbb{P T}-\mathbb{P T}_{\mathbb{R}}$ such that the d-bar operator $\bar{\partial}_{E}$ extends smoothly over $\mathbb{P} \mathbb{T}_{\mathbb{R}}$.

The proof of this is relegated to an appendix. With this, $E$ extends over all of $\mathbb{P T}$ and its restriction to $\mathbb{P T}_{\mathbb{R}}$ has a naturally defined Hermitian metric $H$.

Proof (backward direction): Starting with a pair $(E, H)$, we wish to construct an anti-self-dual Yang-Mills field on $\widetilde{\mathbb{M}}$. We first construct a principal $\mathrm{U}(n)$ bundle $P^{\prime} \rightarrow \widetilde{\mathbb{M}}$ whose fibre $P_{x}^{\prime}$ at $x \in \widetilde{\mathbb{M}}$ is

$$
P_{x}^{\prime}=\left\{\text { holomorphic frames } g \text { of } E \rightarrow D_{x} \mid g \text { is unitary w.r.t. } H \text { on } \partial D_{x}\right\}
$$

In order to see that this is well defined, we first choose a Stein neighbourhood $U$ of $D_{x}$ in $\mathbb{P} \mathbb{T}$ and choose a holomorphic trivialization of $E$ over $U$. By an abuse of notation, denote by $g$ and $H$ the matrices representing the frame $g$ and Hermitian metric $H$ in this trivialisation of $E$. Then $g(x, Z)$ must be holomorphic on $D_{x}$ and satisfy

$$
g H g^{*}=1
$$

on $\partial D_{x}$. This is a Birkhoff factorization of $H$ as $g^{*}$ extends naturally to a holomorphic function on $\mathbb{C} L_{x}-D_{x}$ since complex conjugation on $\mathbb{P} \mathbb{T}$ restricts to $\mathbb{C} L_{x}$ sending $D_{x}$ to $\mathbb{C} L_{x}-D_{x}$. In order for $g$ to be well defined we first need to know that this Birkhoff factorization always has trivial homomorphism factor from $\partial D_{x} \rightarrow \operatorname{GL}(n)$ (i.e., the matrix $\left.H\right|_{\partial D_{x}}$ to be factorized always lies in the 'big cell'). This follows from the fact that $H$ is positive definite, see Gohberg \& Krein (1958) or Mason \& Woodhouse (1996) proposition 9.3.6. Thus, a Birkhoff factorization $g H \tilde{g}=1$ exists for some $g, \tilde{g}$ holomorphic on $D_{x}$ and $\mathbb{C} L_{x}-D_{x}$ respectively, unique up to $g, \tilde{g} \rightarrow A g, \tilde{g} A^{-1}$ for some constant matrix $A$. We need to show that we can choose $A$ so that $\tilde{g}=g^{*}$. We first note that since $H$ is Hermitian, we have that $\tilde{g}^{*} H g^{*}=1$. Therefore, eliminating $H$, on $\partial D_{x}$ we have $g \tilde{g}^{*-1}=\tilde{g}^{-1} g^{*}$. However, the left hand 
side of this equation can be continued holomorphically over $D_{x}$, whereas the right continues holomorphically over $\mathbb{C} L_{x}-D_{x}$, so together they define a matrix valued function that is global on the Riemann sphere, and hence constant by Liouville's theorem. Under $g, \tilde{g} \rightarrow A g, \tilde{g} A^{-1}$ we have $g \tilde{g}^{*-1} \rightarrow A g \tilde{g}^{*-1} A^{*}$ and, since $g \tilde{g}^{*-1}$ is Hermitian, $A$ can be chosen to reduce $g \tilde{g}^{*-1}$ to the identity with the residual freedom of $A$ such that $A A^{*}=1$, i.e., the unitary group. Therefore, $P^{\prime} \rightarrow \widetilde{\mathbb{M}}$ is well defined and naturally has the structure of a principal $U(n)$ bundle.

We now wish to construct an ASDYM connection on $P^{\prime}$. By construction, there are natural trivialisations of $P^{\prime}$ over the $\alpha$-planes in $\widetilde{\mathbb{M}}$ obtained by choosing, for $Z \in \mathbb{P T}_{\mathbb{R}}$, a unitary frame $g_{Z}$ of $E_{Z}$, and requiring that, for each $x$ with $Z \in \partial D_{x}$, $g(x, Z)=g_{Z}$.

Lemma 3.2 There exists a unique connection on $P$ up to gauge transformations such that the above frames are be covariantly constant. Such a connection is necessarily anti-self-dual.

We construct the connection on $P$ using $g$ as follows. The expression $g^{-1} \pi^{A^{\prime}} \nabla_{A A^{\prime}} g$ is holomorphically defined as a section of $\mathcal{O}(1)$ over each $D_{x}$ and is skew-hermitian on $\partial D_{x}$ since $\pi^{A^{\prime}} \partial_{A A^{\prime}}$ is real and $g$ is unitary there. (Here $\mathcal{O}(1)$ is the dual of the tautological line bundle $\mathbb{S} \rightarrow \mathbb{P S}$ over the projective spin bundle; on each $\mathbb{C P}^{1}$ fibre of $\mathbb{P S}$, it restricts to the standard line bundle of Chern class 1 whose sections can be represented by homogeneous functions of degree 1 ). It is therefore equal to $A_{A A^{\prime}} \pi^{A^{\prime}}$ for some skew-Hermitian $A_{A A^{\prime}}$ depending only on $x \in U^{\prime}$ by an extension of Liouville's theorem. The extension of Liouville's theorem in question states that a holomorphic function on the unit disc that is real on the boundary is necessarily a real constant. This follows from the standard Liouville theorem by extending the function over the whole complex plane by setting $f(z)=\overline{f(1 / \bar{z})}$ and noting that the resulting function is continuous and hence holomorphic on $|z|=1$ since it is real there and so is a bounded holomorphic function on $\mathbb{C}$. This extends to sections of $\mathcal{O}(1)$ by considering $\left(\pi_{0^{\prime}}\right)^{-1} g^{-1} \pi^{A^{\prime}} \nabla_{A A^{\prime}} g$ which has a simple pole at $\pi_{0^{\prime}}=0$, but is otherwise, via the above argument, holomorphic on the Riemann sphere and is therefore equal to a skew Hermitian $\left(\pi_{0^{\prime}}\right)^{-1}\left(A_{A 0^{\prime}} \pi_{1^{\prime}}-A_{A 1^{\prime}} \pi_{0^{\prime}}\right)$ where $A_{A A^{\prime}}$ depends only on $x$. $\square$

In the case that $H$ is induced by an anti-holomorphic map $\tilde{\sigma}_{E}: E \rightarrow \bar{E}^{*}$ covering the standard complex-conjugation, the ASDYM field can be constructed directly on $\mathbb{M}$ via the standard Ward transform and the fact that it will give rise to a real ASDYM field follows from the reality structure $\tilde{\sigma}_{E}$. The construction works by defining, for $x \in \mathbb{M}, E_{x}^{\prime}=\Gamma\left(\mathbb{C} L_{x}, E\right)$ with Hermitian form induced by $\tilde{\sigma}_{E}$. The construction of the connection follows as above replacing $g$ by the expression for a frame of $E_{x}^{\prime}=\Gamma\left(\mathbb{C} L_{x}, E\right)$ that is unitary on the real slice in some local trivialisation of $E \rightarrow \mathbb{P T}$ on a neighbourhood of $D_{x}$.

\section{Remarks.}

1. This theorem can easily be extended to any compact gauge group $G$ by embedding $G$ in $\mathrm{U}(n)$ for some $n$. The construction is most easily stated in terms of principal bundles as in the introduction:

Theorem 3 Gauge equivalence classes of $C^{\infty}$ solutions to the ASDYM equations with gauge group $G$ are in 1-1 correspondence between principal $G_{\mathbb{C}}$ bundles $P \rightarrow \mathbb{P T}$ together with a $C^{\infty}$ reduction of the structure group to $G$ over $\mathbb{P T}_{\mathbb{R}}$ where $G_{\mathbb{C}}$ is 
the complexification of $G$. The reduction of the structure group over $\mathbb{P} \mathbb{T}_{\mathbb{R}}$ can be alternatively expressed as a $C^{\infty}$ section $H: \mathbb{P}_{\mathbb{R}} \rightarrow P / G$ of the $G_{\mathbb{C}} / G$ bundle $P / G$.

2. Unlike the case of Euclidean signature, solutions to equations in indefinite signature can have pretty much arbitrarily low regularity. It is clear that, taking the correspondence in the reverse direction, the bundle $P$ or $E$ over $\mathbb{P T}$ is always necessarily analytic by elliptic regularity, but $H$ can be chosen to have any regularity for which the Birkhoff factorization will work. If we work in a Hölder space framework, the regularity of solutions to the Birkhoff factorization problem have regularity $C^{k, \alpha^{\prime}}$ when $H$ has regularity $C^{k, \alpha}$ for $\alpha^{\prime}<\alpha$ assuming that there are no jumping lines (as is the case here). This follows from the corresponding results for the Hilbert transform and the implicit function theorem. The connection on space-time is therefore of regularity $C^{k-1, \alpha^{\prime}}$ since one further derivative is taken. This suggests that given an ASDYM connection on space-time of given regularity, $H$ can be shown to have one extra degree of regularity. However, the construction of $H$ from teh connection in the proof loses many degrees of regularity so these methods will not yield a definitive theorem.

\subsection{The topology of $E$}

The theorem implies that any holomorphic vector bundle $E \rightarrow \mathbb{P T}$ with hermitian metric $H$ on $\left.E\right|_{\mathbb{P T}_{\mathbb{R}}}$ will give rise to anti-self-dual Yang-Mills fields with gauge group $\mathrm{U}(n)$ so long as the hermitian metric $H$ is positive definite - we do not need to concern ourselves with 'jumping lines'.

The topology of holomorphic vector bundles on $\mathbb{C P}^{3}$ are characterised by the Chern classes $c_{1}, c_{2}$ and $c_{3}$ except in the case of rank 2 bundles for which $c_{3}$ is trivial, but when $c_{1}$ is even, can admit a mod 2 ' $\alpha$-invariant,' Atiyah \& Rees (1976). All such invariants can be non-trivial in contradistinction with the case of instantons on $S^{4}$, for which the only possible non-trivial topological invariant is the second Chern class of the original Yang-Mills vector bundle on $S^{4}$ which is the same as the second Chern class of $E \rightarrow \mathbb{C P}^{3}$. Here $c_{1}$ and $c_{3}$ can be non-trivial also.

Firstly $c_{2}\left(E^{\prime}\right)(\widetilde{\mathbb{M}})=2 c_{2}(E)\left(\mathbb{C P}^{2}\right)$ since $c_{2}\left(E^{\prime}\right)(\widetilde{\mathbb{M}})$ can be represented as the integral of $\left(-1 / 8 \pi^{2}\right) \operatorname{tr} F^{2}$ over $\widetilde{\mathbb{M}}$. This integral is the same as that over the quadric $\sum_{\alpha}\left(Z^{\alpha}\right)^{2}=0$ in $\mathbb{P T}$ since this is a section of the fibration $\mathscr{F}-\partial \mathscr{F}=\mathbb{P T}-\mathbb{P} \mathbb{T}_{\mathbb{R}} \rightarrow \widetilde{\mathbb{M}}$ (this geometry is explained more fully in appendix 2). However, the quadric is twice the generator of the 2nd cohomology of $\mathbb{P} \mathbb{T}$.

Furthermore non-trivial $c_{1}(E)$ is also allowed. This arises from $c_{1}\left(E^{\prime}\right)$ since $c_{1}(E)[$ line $]=c_{1}\left(E^{\prime}\right)[\beta$-plane $] / 2$ using the identification in the previous paragraph since the $\beta$-plane becomes identified with a conic obtained by intersecting a plane in $\mathbb{P T}$ with the quadric. Note that since $E^{\prime}$ is flat on $\alpha$-planes, $c_{1}\left(E^{\prime}\right)[\alpha$-plane $]=0$ so the possible first Chern classes are measured just by evaluation on $\beta$-planes and must be even from above. $\mathrm{U}(1)$ examples with nontrivial $c_{1}$ will be given in the next section.

More remarkably $c_{3}(E)$ can be non-trivial. For general gauge group there is a mod 2 relation on $c_{3}$, i.e., if $c_{1}=c_{2}=0, c_{3}$ must be even (Rees private communication), although for $\mathrm{SU}(2)$ there is only the $\alpha$-invariant. At least in the $\mathrm{SU}(2)$ case it is known that this invariant can be non-trivially realised with a holomorphic vector bundle for any given even $c_{1}$ and arbitrary $c_{2}$, for example it is non-trivial 
for $E=\mathcal{O}(2) \oplus \mathcal{O}(-2)$ (although, of course, $c_{2}$ is also non-trivial for this bundle), Atiyah \& Rees (1976).

Non-trivial $c_{3}$ cannot arise from the topology of $E^{\prime} \rightarrow \widetilde{\mathbb{M}}$ as $\widetilde{\mathbb{M}}$ is 4-dimensional and indeed, non-trivial third Chern classes can arise when $E^{\prime}$ is trivial by choosing $c_{1}(E)=c_{2}(E)=0$ but $c_{3}(E) \neq 0$. To see where the topological non-triviality comes from in this case, we note that for unitary groups, $\left.E\right|_{\mathbb{P}_{\mathbb{R}}}$ will be trivial so that a trivialization of $\left.E\right|_{\mathbb{P}_{\mathbb{R}}}$ can be pulled back to $\mathscr{F} \mathbb{R}$ and compared to the pullback of a trivialisation $E^{\prime}$. The gauge transformation between these trivializations gives a map $g: \mathscr{F}_{\mathbb{R}} \rightarrow \mathrm{U}(n)$ and this will be topologically non-trivial when $c_{3}(E)$ or $\alpha$ are non trivial. ${ }^{5}$

\subsection{The case when $E$ is trivial}

For a 'small' ASDYM field, the topological invariants of $E$ will necessarily be trivial and this implies that $E$ is analytically trivial. If $E$ is trivial as a holomorphic vector bundle, a holomorphic trivialization of $E$ is unique up to a global constant $\operatorname{GL}(n, \mathbb{C})$ transformation. In such a global trivialisation, $H: \mathbb{R P}^{3} \rightarrow \mathrm{Herm}_{n}^{+}$where $\mathrm{Herm}_{n}^{+}$ denotes the space of $n \times n$ positive definite Hermitian matrices and $H$ is defined up to $H \rightarrow g H g^{*}$ for constant $g \in \mathrm{GL}(n, \mathbb{C})$. Such equivalence classes of $H$ completely characterise ASDYM fields on $S^{2} \times S^{2}$ with 'small' data. The correspondence between $H$ and the corresponding ASDYM field is a nonlinear analogue of the X-ray transform (see below).

\section{Examples}

\subsection{The abelian case}

Consider first the case where $E$ is the trivial line bundle. Then $H$ is simply a real non-vanishing function on $\mathbb{P T}_{\mathbb{R}}$ and the problem of constructing the corresponding field on space-time proceeds by means of standard twistor integral formulae with twistor function log $H$ that go back to Ward and Sparling, see Ward (1977). In the affine coordinates given before, the integral formula leads to the following standard formula for the ASD Maxwell field

$$
\phi_{A B}(x)=\oint_{\omega^{A}=x^{A A^{\prime}} \pi_{A^{\prime}}} \frac{\partial^{2} \log H}{\partial \omega^{A} \partial \omega^{B}} \pi_{C^{\prime}} \mathrm{d} \pi^{C^{\prime}} .
$$

It is worth noting that because $H^{1}(\mathbb{P T}, \mathcal{O})$ is trivial, there are no ASD Maxwell fields on $\mathbb{M}$. The Maxwell fields obtained from the construction above are odd under the antipodal map on $\widetilde{\mathbb{M}}$ since the integral above requires an orientation on the line $L_{x}$.

A further novelty is that $H$ is a smooth function on $\mathbb{P T}_{\mathbb{R}}$ unique up to a constant rather than an element of a cohomology class which would be the norm for a Penrose transform. The transform here yields a helicity shifted variant of the standard X-ray transform.

Naively, we can connect the $f=\log H$ with cohomology following Atiyah (1979). Take $f$ to be analytic and extend it to some neighbourhood $U$ of $\mathbb{P T}_{\mathbb{R}}$. On a

\footnotetext{
${ }^{5}$ It was erroneously assumed that these classes should vanish in Mason (1995).
} 
neighbourhood $V$ of a line that is divided into two parts by $U$, say $V=V_{0} \cup V_{1}$ with $V_{0} \cap V_{1}=U \cap V$, it can be taken to be the Cech representative for a cohomology class relative to that covering of $V$. (Alternatively a Dolbeault representative can be obtained by extending $f$ off $U$ as a smooth but non-holomorphic function on $\mathbb{P T}$ and, on a neighbourhood $V$ of a line, we can consider the dolbeault form $\alpha$ such that $\alpha=0$ on $V_{0}$ and $\alpha=\bar{\partial} f$ on $V_{1}$.) Clearly such descriptions fail globally since, as the space-time point does a circuit around a null geodesic in $\mathbb{M}$, if we follow this path with different choices of $V, V_{0}$ and $V_{1}$ will be interchanged and so $f$ would have to be identified with $-f$. This construction also fails to explain how the cohomological gauge freedom is fixed.

One way to understand the construction globally and cohomologically is as follows. First, if such a function $f=\log H$ defined up to a constant is taken to be analytic, it can be thought of as a relative cohomology element in $H_{\mathbb{P T}-U}^{1}(\mathbb{P T})$ where $U$ is some small open neighbourhood of $\mathbb{P} \mathbb{T}_{\mathbb{R}}$ via the connecting homomorphism of the long exact relative cohomology sequence

$$
\ldots \rightarrow H^{1}(\mathbb{P} \mathbb{T}) \rightarrow H_{\mathbb{P T}-U}^{1}(\mathbb{P T}) \stackrel{\delta}{\rightarrow} H^{0}(U) \rightarrow H^{0}(\mathbb{P} \mathbb{T}) \rightarrow \ldots
$$

using the observation that $H^{1}(\mathbb{P} \mathbb{T})=0$ and $H^{0}(\mathbb{P} \mathbb{T})=$ constants. The relative cohomology then has a natural pairing with each $\left(D_{x}, \partial D_{x}\right)$ which is realised by the usual integral formulae. This ties in with a point of view developed in Mason (1995) in which the twistor space is taken to be the non-Hausdorff space obtained by gluing together two copies of $\mathbb{P T}$ along $U$. Cohomology on the space is given by the relative cohomology group $H_{\mathbb{P T}-U}^{1}(\mathbb{P} T)$ by a result of Bailey (1985), see also $\S 6$ of Mason \& Hughston (1990).

The detailed correspondence between the Penrose transform and the X-ray transform has been much studied elsewhere, Woodhouse (1992), Mason (1995), Sparling (1998), Bailey, Eastwood, Gover and Mason (1999, 2003), Bailey \& Eastwood (2001). This connection between the Penrose transform and X-ray transform is also used in twistor string theory, Witten (2004) and Berkovits and Witten (2004).

The case where $E$ is nontrivial: If $E$ is a nontrivial line bundle, it must be isomorphic to $\mathcal{O}(k)$ for some $k$. We must then take $H$ to be a (real) non-vanishing section of $\mathcal{O}(-2 k)$ over $\mathbb{P T}_{R}$. Taking the simplest case, $k=-1$, we can set $H=$ $\sum_{\alpha}\left(Z^{\alpha}\right)^{2}$. This can be transformed explicitly to yield the $\mathrm{U}(1)$ gauge field with curvature

$$
F=\frac{2 \mathrm{~d} w_{1} \wedge \mathrm{d} \bar{w}_{1}}{\pi\left(1+\left|w_{1}\right|^{2}\right)^{2}}+\frac{2 \mathrm{~d} w_{2} \wedge \mathrm{d} \bar{w}_{2}}{\pi\left(1+\left|w_{2}\right|^{2}\right)^{2}}
$$

This gives rise to a gauge field with non-trivial first Chern class on $\widetilde{\mathbb{M}} \cdot{ }^{6}$ Clearly the metric $\mathrm{e}^{f} H^{-k}$ on $\mathcal{O}(k)$ will give the sum of $-k$ times the above solution with that described above using the standard twistor integral formula for $f$.

\subsection{The t'Hooft and Ward ansatze}

Examples of non-abelian ASDYM fields with gauge group $\operatorname{SL}(2, \mathbb{R})$ on $\widetilde{\mathbb{M}}$ are constructed from the t'Hooft ansatze, with $E=\mathcal{O}(1) \oplus \mathcal{O}(-1)$ in $\S 10.5 .2$ of Mason \&

\footnotetext{
${ }^{6}$ This is an analytic continuation of the ASD Coulomb/Dirac monopole solution centred on the complex curves either $\mathbf{x}=0=\mathbf{y} \cdot \mathbf{y}$ or $\mathbf{y}=0=\mathbf{x} \cdot \mathbf{x}$-the points of these curves correspond to the complex lines in $\mathbb{P T}$ that generate the given quadric, see $\S$ I.6.2 of Hughston \& Mason (1990).
} 
Woodhouse (1996).

It is not so easy to encode the reality conditions for real $\mathrm{SU}(2)$ solutions with the t'Hooft ansatz (see, for example, the next section). Imposing a symmetry on $\widetilde{\mathbb{M}}$ along $\operatorname{Im} w_{1} \partial / \partial w_{1}$ reduces the ASDYM equations to a Yang-Mills-Higgs system on $2+1$ de Sitter space that has been studied by Kotecha \& Ward (2001). The specific solution actually considered by Kotecha \& Ward is not smooth when pulled back to $\widetilde{\mathbb{M}}$ (thus there is also a singularity at infinity in $2+1$ de Sitter space), but the ansatz can be adapted to give smooth solutions on $\widetilde{\mathbb{M}}$.

Reformulating $\S 7$ of Kotecha \& Ward (2001) but without imposing the symmetry, we consider the case where $E=\mathcal{O}(k) \oplus \mathcal{O}(-k)$ and

$$
H=\left(\begin{array}{cc}
2 Q^{-1} \cosh f & \mathrm{e}^{-f} \\
\mathrm{e}^{-f} & Q \mathrm{e}^{-f}
\end{array}\right)
$$

where $Q \in H^{0}(\mathbb{P} \mathbb{T}, \mathcal{O}(2 k))$ is a polynomial of homogeneity $2 k$ that does not vanish on $\mathbb{P T}_{\mathbb{R}}$ and $f$ is a smooth function on $\mathbb{P} \mathbb{T}_{\mathbb{R}}$. A straightforward choice of $Q$ is $Q=\left(\sum_{\alpha}\left(Z^{\alpha}\right)^{2}\right)^{k}$ This can be used to give a solution by means of the Ward ansatze as described in $\S 8.2 .4$ of Ward \& Wells (1990): we note that

$$
H=F R, \quad \text { where } \quad F=\left(\begin{array}{cc}
\mathrm{e}^{f} & 2 Q^{-1} \cosh f \\
0 & \mathrm{e}^{-f}
\end{array}\right) \quad \text { and } \quad R=\left(\begin{array}{cc}
0 & -1 \\
1 & Q
\end{array}\right)
$$

and that $R: \mathcal{O}(k) \oplus \mathcal{O}(-k) \rightarrow \mathcal{O}(-k) \oplus \mathcal{O}(k)$ is a global map of vector bundles on $\mathbb{P T}$. So, as far as the Birkhoff factorization is concerned, we are reduced to an example of the Ward ansatze as detailed in $\S 8$ of Ward and Wells (1990) for which the reconstruction of the space-time ASDYM field can be implemented by quadratures. ${ }^{7}$ The full calculations are performed in appendix $\$$ B.4 where a more detailed formalism is established that expedites the calculations.

\subsection{Split signature instantons}

The case in which $H$ is induced from a reality structure on $E$ is in effect the case where the solution on $\widetilde{\mathbb{M}}$ is pulled back from one on $\mathbb{M}$ that is constructed from a global holomorphic vector bundle over $\mathbb{P} \mathbb{T}$ using the standard Ward construction. This is what we will mean by a 'split signature instanton'- there is no analogue of the Bogomolny bound for the action by the second Chern class in split signature, and so the concept of an instanton is not well defined, but these solutions are the ones that are defined purely algebraically geometrically.

This case reduces to the standard Ward construction in which, in order to obtain a regular solution on $\mathbb{M}, E$ must be trivial on restriction to the complex lines that are complexifications of real lines. This non-singularity is in any case a consequence of the assumption that the reality structure on $E$ induces a positive-definite hermitian structure on the restriction of $E$ to the real slice since the triviality of $E$ over $L_{x}$ follows by the same argument as given in the reconstruction of the ASDYM field from the bundle. This in particular implies that $c_{1}(E)=0$. Furthermore $E$ must be semi-stable since if there is a destabilizing subsheaf, it will agree with $\mathcal{O}(k)$,

\footnotetext{
${ }^{7}$ The Kotecha Ward solution has $k=1, Q$ given as above, and $f=\log Q /\left(\left(Z^{2}\right)^{2}+\left(Z^{3}\right)^{2}\right)$, but $f$ is not smooth on all of $\mathbb{P T}_{\mathbb{R}}$ and so the pullback of the corresponding solution to $\widetilde{\mathbb{M}}$ is not smooth.
} 
$k>0$, on a generic line and this will lead to $E$ being non-trivial on a generic real line. It is easy to see that if it is semistable but not stable, the subsheaf generically isomorphic to $\mathcal{O}(0)$ will give rise to a line subbundle of $E^{\prime}$ on space-time on which the connection is trivial and so the connection will be reducible. Thus, assuming the ASDYM connection on $E^{\prime}$ is not reducible, we may assume $E$ to be stable and the ADHM machinery may be invoked to construct $E$ and the solution on space-time as described in Atiyah (1979).

Theorem 4 Split signature instantons necessarily have even $c_{2}(E)$ and exist for $c_{2}(E)=2$.

Proof: In complexified Minkowski space, $\mathbb{C M}$ there is a hypersurface $\Sigma$ on which instantons are singular (points of $\Sigma$ correspond to lines in $\mathbb{C P}^{3}$ on which $E$ fails to be trivial) and we must choose our bundle $E$ so that, not only are the fields real, but also this hypersurface has no real points. The singular hypersurface has degree $c_{2}(E)$ in $\mathbb{P}^{5}$ and determines $E$ at least for $c_{2}(E)=1$ or 2 , Hartshorne (1978). In the case $c_{2}(E)=1$, the hypersurface is linear, and must be real for a real solution. It is easy to see that it must then intersect $\mathbb{M}$ and indeed this will be the case for any odd $c_{2}(E)$ as an algebraic hypersurface of odd degree will always have real points.

For $c_{2}(E)=2$, the singular hypersurface $\Sigma$ is a quadratic cone on $\mathbb{C P}^{5}$ of rank 3 subject to a certain 'Poncelet' condition, Hartshorne (1978). For reality, the 3-plane in the kernel of this quadratic form is the complexification of a real $\mathbb{R}^{3} \subset \mathbb{R}^{6}$ and the quadric defining $\mathbb{M}$ restricts to be either Lorentzian or of definite sign on this plane. The former cannot lead to smooth solutions, so we focus on the latter in the following. In terms of our homogenous coordinates $(\mathbf{x}, \mathbf{y}) \in \mathbb{R}^{3} \times \mathbb{R}^{3}$ on $\mathbb{R P}^{5}$ we can, after an $\mathrm{SO}(3,3)$ transformation, express $\Sigma$ as $S(\mathbf{x}, \mathbf{x})=\mathbf{x} \cdot \mathbf{x}$ for some symmetric trace-free matrix $S$. The 'Poncelet condition' of the conic $S(\mathbf{x}, \mathbf{x})=\mathbf{x} \cdot \mathbf{x}$ with respect to the conic $\mathbf{x} \cdot \mathbf{x}=0$ requires that for an arbitrary point $\mathbf{x}_{0}$ on $\mathbf{x} \cdot \mathbf{x}=0$, if we construct the tangent $l_{0}$ at $\mathbf{x}_{0}$ and its intersections with $S(\mathbf{x}, \mathbf{x})=\mathbf{x} \cdot \mathbf{x}$ at points $\mathbf{a}_{\mathbf{1}}$ and $\mathbf{a}_{\mathbf{2}}$, then the other tangents to $\mathbf{x} \cdot \mathbf{x}=0$ through $\mathbf{a}_{\mathbf{1}}$ and $\mathbf{a}_{\mathbf{2}}$ must meet on the conic $S(\mathbf{x}, \mathbf{x})=\mathbf{x} \cdot \mathbf{x}$. This Poncelet condition is satisfied iff the $\operatorname{tr}\left(S^{2}\right)=3 / 2$. It is clear that this can be satisfied in such a way that $\Sigma$ has no real points, i.e., with the eigenvalues of $S$ less than 1 .

Although these solutions can all be constructed explicitly by means of the t'Hooft ansatze based on a solution to the ultrahyperbolic wave equation $\phi=\sum_{i=1}^{3} \lambda_{i} /(x-$ $\left.x_{i}\right)^{2}$, the $x_{i}$ must be three points of the conic $\mathbf{x} \cdot \mathbf{x}=0$ tangent to the sides of a Poncelet triangle as described above, and so cannot be real. Therefore $\phi$ cannot be real and it will be difficult to identify the values of $x_{i}$ and $\lambda_{i}$ that give rise to a real solution or represent it in a real (unitary) gauge.

Instead, the ADHM construction is worked through in an appendix to give an explicit formula for the connection (it is not presented here as it requires a description of the twistor correspondence that is only developed earlier in the appendix).

\section{Nonlinear scattering theory}

In this section we construct the map from initial data at $-\infty$ to final data at $+\infty$ at least in the context of small data giving rise to small solutions (so that the topology of both $E$ and $E^{\prime}$ can be taken to be trivial). The key idea rests on two facts. 
Firstly the intersections of $\alpha$-planes in space-time with null infinity are circles, and the flatness condition on $\alpha$-planes implies that the holonomy around these circles must be trivial. Secondly, we find that the characteristic data on past null infinity can be encoded into holonomies in effect around two halves of such circles, one associated to the part of the $\alpha$-plane in past null infinity and the other in a fixed family of $\alpha$-planes associated to the ' $t=0$ ' hypersurface dividing past from future null infinity. The scattering map must then take these holonomies to their inverse on the corresponding intersection of the $\alpha$-plane with future null infinity. We need to prove however that these holonomies uniquely determine and are determined by the initial data, the final data and a compatible ASDYM field on $\mathbb{M}$, at least for small data.

The construction will involve taking the family of holonomies of the connection and performing a sequence of two Birkhoff factorizations on the holonomies, the first to find $H$ (which determines the solution on all of $\widetilde{\mathbb{M}}$ ) and the second to find the final data. The sense in which the data is small is that for the corresponding twistor data, the holomorphic vector bundle $E$ is trivial so that the solution is encoded into $H$. It is also small in the sense that these Birkhoff factorizations do not jump. This can be expressed as an analytic smallness condition on the asymptotic characteristic data, see Novikov (2002) for an explicit statement of the smallness condition. These calculations are nevertheless fully nonlinear.

We will see that the the calculation can be performed on restriction to certain 2 -planes, $\beta$-planes, on the initial and final data surface $\mathscr{I}$, so there is no interaction between data posed on one $\beta$-plane and another.

\subsection{The geometry}

In split signature, the global structure does not provide a canonical decomposition into past and future since there are two time-like directions and we must make an (unnatural) choice of one of them to proceed. This is possible, and we will see that we can define scattering in this way in spite of the signature.

The choice of infinity in this conformally invariant context is also arbitrary and we do this first. We choose the point $i^{-} \in \widetilde{\mathbb{M}}$ at past infinity and denote its antipode by $i^{+}$, future infinity. The data at infinity will be posed on the lightcone $\mathscr{I}$ of $i^{-}$ which reconverges on $i^{+}$. In order to make a choice of past and future, we choose a linear hyperplane $\Sigma$ in $\mathbb{R P}^{5}$ that is not tangent to $Q=0$, and separates $i^{+}$from $i^{-}$ cutting $\mathscr{I}$ into $\mathscr{I}^{ \pm}$.

In the coordinatisation $\left(w_{1}, w_{2}\right)$ of $\widetilde{\mathbb{M}}$ of equation (11), the points $i^{-}$and $i^{+}$can be taken to be $w_{1}=w_{2}=0$ and $w_{1}=w_{2}=\infty$ respectively. The light cone $\mathscr{I}$ of $i^{-}$ is the hypersurface $\left|w_{1}\right|=\left|w_{2}\right|$ and reconverges at $i^{+}$. We can coordinatise $\mathscr{I}$ with $\left(w_{1}, \eta\right) \in \mathbb{C} \times S^{1}$ by setting

$$
\left(w_{1}, w_{2}\right)=\left(w_{1}, \mathrm{e}^{i \eta} w_{1}\right)
$$

$\mathscr{I}$ divides $\widetilde{\mathbb{M}}=S^{2} \times S^{2}$ into two copies $\mathbb{M}^{ \pm}$of affine $\mathbb{R}^{4}$ and we take $\mathbb{M}^{+}=$ $\left\{\left(w_{1}, w_{2}\right) ;\left|w_{1}\right|>\left|w_{2}\right|\right\}$ as physical space-time and discard $\mathbb{M}^{-}$. A convenient choice for $\Sigma$ is the hyperplane $x_{3}=0$ or equivalently $\left|w_{1}\right|=1$ (i.e., $x_{3} /\left(x_{3}-y_{3}\right)$ is taken to be the time variable). $\Sigma$ divides $\mathscr{I}$ into $\mathscr{I}^{ \pm}=\left\{ \pm\left(\left|w_{1}\right|-1\right) \geq 0\right\}$ with $\mathscr{I}^{-}$being past null infinity and $\mathscr{I}^{+}$future null infinity. The antipodal map on $\widetilde{\mathbb{M}}$ sends $\mathscr{I}$ to itself giving a canonical identification between $\mathscr{I}^{+}$and $\mathscr{I}^{-}$(the light-cone of a 
point of $\mathscr{I}^{-}$reconverges on the 'antipodal' point on $\mathscr{I}^{+}$) and so we will be able to compare initial data on $\mathscr{I}^{-}$to 'final data' on $\mathscr{I}^{+}$.

\subsection{The characteristic data}

Characteristic data for the ASDYM equations is a connection $A$ on a bundle $E^{\prime}$ over $\mathscr{I}^{-}$that is flat on the $\alpha$-planes on $\mathscr{I}^{-} .8$ Taking a parallel propagated frame of $E^{\prime}$ from $i^{-}$up the generators of $\mathscr{I}^{-}$will therefore yield a gauge in which $A^{-}=$ $A^{-}\left(w_{1}, \eta\right)\left(\mathrm{d} \eta+i w_{1} \mathrm{~d} \bar{w}_{1}-i \bar{w}_{1} \mathrm{~d} w_{1}\right)$ and this $A^{-}\left(w_{1}, \eta\right)$ will be a smooth function on $\mathscr{I}^{-}$with values in $\operatorname{su}(n)$ that vanishes at $i^{-}$. We must also require that the holonomy of $A^{-}$about the unit circle in each $w_{1}$ plane (of fixed $\eta$ ) should vanish as these circles bound $\alpha$-planes in $\mathbb{M}^{+}$on which the connection must be flat. The function $A^{-}\left(w_{1}, \eta\right)$ is otherwise freely prescribable. To avoid some technicalities, we will, however, assume that $A^{-}\left(w_{1}, \eta\right)$ vanishes at $\Sigma \cap \mathscr{I}$ (i.e., at $\left|w_{1}\right|=1$ ) and near $i^{-}{ }^{9}$ Our aim is to show that $A^{-}$gives rise to a unique solution to the ASDYM equations on $\mathbb{M}^{+}$and find a procedure to determine the 'final' data on $\mathscr{I}^{+}$.

\section{$5.3 \quad \beta$-planes and their twistor theory}

The $\beta$-planes on $\mathscr{I}$ are given by $w_{1}=\mathrm{e}^{i \eta} w_{2}$ and denoted $W_{\eta}^{\prime}$; they foliate $\mathscr{I}-i^{ \pm}$. All $W_{\eta}^{\prime}$ intersect at $i^{ \pm}$but are otherwise disjoint and are topologically 2-spheres with complex stereographic coordinate $w_{1}$. The hypersurface $\Sigma$ cuts $W_{\eta}^{\prime}$ into the two discs $W_{\eta}^{\prime \pm}=\left\{W_{\eta}^{\prime}, \pm\left(\left|w_{1}\right|-1\right) \geq 0\right\}$.

The correspondence with twistor space $\mathbb{P T}$ is as follows. The points $i^{ \pm}$correspond in twistor space to a real line $I$ (the \pm corresponding to the choices of orientation of $I$ ). Each $\beta$-plane $W_{\eta}^{\prime}$ on $\mathscr{I}$ is dual to a 2-plane $W_{\eta} \subset \mathbb{P T}$ that contains $I$. Given $Z \in \mathbb{P} \mathbb{T}-I$, the corresponding $\alpha$-plane $\alpha_{Z} \subset \widetilde{\mathbb{M}}$ intersects $\mathscr{I}$ in a null geodesic that lies in a unique $W_{\eta}^{\prime}$ as a great circle $Z^{\prime}=\alpha_{Z} \cap W_{\eta}^{\prime}\left(=\alpha_{Z} \cap \mathscr{I}\right)$ and we define $W_{\eta}$ to consist of those $Z$ such that $\alpha_{Z} \cap \mathscr{I} \subset W_{\eta}^{\prime}$. A point $p \in W_{\eta}^{\prime}$ corresponds to the line in the projective plane $W_{\eta}$ consisting of those $Z^{\prime}$ through $p$; thus $W_{\eta}$ and $W_{\eta}^{\prime}$ modulo the antipodal map (which reduces $W_{\eta}^{\prime}$ from $S^{2}$ to $\mathbb{R P}^{2}$ ) are in projective duality.

We can realise this in coordinates as follows. Given homogenous coordinates $z_{i}$, $i=1,2,3$ on $W_{\eta}$, the corresponding null geodesic on $W_{\eta}^{\prime}$ can be taken to be the great circle represented in terms of the stereographic coordinate $w_{1}$ as

$$
z_{3}\left(1-\left|w_{1}\right|^{2}\right)+\Re\left(\left(z_{1}+i z_{2}\right) w_{1}\right)=0 .
$$

This gives $I$ as the line $z_{3}=0$. The intersection $\Sigma_{\eta}=\Sigma \cap W_{\eta}^{\prime}=\partial W_{\eta}^{\prime \pm}$ is a null geodesic corresponding to the point $Z_{\Sigma_{\eta}} \in W_{\eta}$ with homogeneous coordinates $(0,0,1)$. Thus, a general real twistor $Z \in \mathbb{P T}_{\mathbb{R}}-I$ can be parametrized by $Z=\left(z_{i}, \eta\right)$ where $z_{i} \sim \lambda z_{i}, \lambda \in \mathbb{R}^{*}$ (globally this is a blowup of $I$ in $\mathbb{P T}_{\mathbb{R}}$ ).

Let $\widetilde{W}_{\eta}$ be the double cover of $W_{\eta}$. It can be represented as the unit sphere $\sum z_{i}^{2}=1$ and is in duality with the sphere $W_{\eta}^{\prime}$ in the sense that points of one corresponds to oriented great circles in the other.

\footnotetext{
${ }^{8}$ On $\mathscr{I}$ the two-surfaces on which $w_{1} w_{2}$ has constant phase are the $\alpha$-planes.

${ }^{9}$ Both conditions are stronger than we need. The first guarantees the vanishing of the holonomy around $\left|w_{1}\right|=1$ and makes certain choices we will make later canonical. The second condition guarantees that $A^{-}$is the restriction of a smooth 1 -form from $\widetilde{\mathbb{M}}$ at $i^{-}$.
} 


\subsection{The scattering on $W_{\eta}^{\prime}$}

In the following, we fix a value of $\eta$, and all our considerations will be concerned with the relationship between initial data $A^{-}$on $W_{\eta}^{\prime-}$, a $\mathrm{U}(n)$ valued matrix function $h\left(z_{i}, \eta\right)$ on $\widetilde{W}_{\eta}$, twistor data $H\left(z_{i}, \eta\right)$ on $W_{\eta}$ and then final data on $W_{\eta}^{\prime+}$.

Given the initial data consisting of the connection $A^{-}$restricted to $W_{\eta}^{\prime-}$, we first fix a covariantly constant frame $f: E^{\prime}=\mathbb{C}^{n}$ of the Yang-Mills vector bundle $E^{\prime}$ around $\partial W_{\eta}^{\prime-}$ (which exists as $A^{-}$has trivial holonomy around $\partial W_{\eta}^{\prime-}$ ).

Definition 5.1 We define $h\left(z_{i}, \eta\right): \widetilde{W}_{\eta} \rightarrow \mathrm{U}(n)$ to be the holonomy around the loop formed by the oriented geodesic in $W_{\eta}^{\prime-}$ corresponding to $\left(z_{i}, \eta\right) \in \widetilde{W}_{\eta}$ and one of the arcs of $\partial W_{\eta}^{\prime-}$ required to close the semicircle (because of the vanishing of holonomy around $\partial W_{\eta}^{\prime-}$, it doesnt matter which arc is chosen).

For $\widetilde{Z} \in \widetilde{W}_{\eta}$, a change in orientation of the corresponding geodesic in $W_{\eta}^{\prime}$ corresponds to $\widetilde{Z} \rightarrow-\widetilde{Z}$ and we will therefore have $h\left(-\widetilde{Z}^{\prime}\right)=h\left(\widetilde{Z}^{\prime}\right)^{-1}$. We also note that as $\widetilde{Z}^{\prime}$ tends towards a semicircle of $\partial W_{\eta}^{\prime-}$, i.e., $z_{1}, z_{2} \rightarrow 0, h \rightarrow I_{n}$.

Definition 5.2 We now define the twistor data $H: \mathbb{P T}_{\mathbb{R}} \rightarrow \operatorname{Herm}_{n}, \mathrm{Herm}_{n}$ being the $n \times n$ hermitian matrices, to be

$$
H:=g^{-*} g^{-}
$$

where $g_{-}$is the solution to the following Birkhoff factorization problem in the $z_{3}$ variable:

$$
h\left(z_{i}, \eta\right) g^{-}\left(z_{i}, \eta\right)=g^{+}\left(z_{i}, \eta\right) .
$$

Here we have fixed a positive scaling of the homogenous coordinates by setting $\left|z_{1}^{2}+z_{2}^{2}\right|=1$ and $g^{ \pm}$extends holomorphically over $\pm \Im z_{3} \geq 0$ in the complex $z_{3}$ plane for each real $z_{1}, z_{2}$, and we normalize $g^{ \pm}$by the condition that, at $z_{3}=\infty$, $h=g^{ \pm}=I_{n}$. $H$ is clearly a positive definite Hermitian matrix function of $\left(z_{i}, \eta\right)$.

Remarks: 1. This is where we use the smallness assumption on the data: the Birkhoff factorization in general exists only when we also allow a factor of $\Delta$ with diagonal entries given by powers of $\left(z_{3}+i\right) /\left(z_{3}-i\right)$, but $\Delta$ is the identity matrix for sufficiently small $h$.

2. We have also used the vanishing of $A^{-}$on $\Sigma \cap \mathscr{I}$ in the normalization conditions for $g^{ \pm}$. Had we not done so, the normalization condition would be more complicated.

Lemma 5.1 $H(-\widetilde{Z})=H(\widetilde{Z})$ so that $H$ is defined on $\mathbb{P T}_{\mathbb{R}}$.

Proof: We first note that $g^{-*} g^{-}=g^{+*} g^{+}$follows from the unitarity of $h$. Furthermore, $h(-\widetilde{Z})=h(\widetilde{Z})^{-1}$ implies that $h(-\widetilde{Z})^{-1} g^{-}(\widetilde{Z})=g^{+}(\widetilde{Z})$ so that $g(-\widetilde{Z})^{ \pm}=$ $g(\widetilde{Z})^{\mp}$ by the uniqueness of the Birkhoff factorization. Thus

$$
H(-\widetilde{Z})=g^{+*} g^{+}=g^{-*} g^{-}=H(\widetilde{Z})
$$

as required.

This $H$ can now be used as in the main theorems to determine an ASDYM field on $\widetilde{\mathbb{M}}$. We need to show that it correctly reproduces the initial data on $\mathscr{I}^{-}$. Clearly we only need to see that it correctly reproduces the appropriate initial data on each $W_{\eta}^{\prime-}$. It is sufficient to prove that the connection that it determines on $W_{\eta}^{\prime-}$ leads to the given holonomy matrix $h\left(z_{i}, \eta\right)$ because of the following theorem: 
Theorem 5 (Manakov \& Zakharov 1981, Novikov 2002) Let $A$ be a twice differentiable $\mathrm{U}(n)$ connection on the projective plane $\mathbb{R P}^{2}$ that vanishes on the line at $\infty, l_{\infty}$ and let $h$ be the $\mathrm{U}(n)$-valued function on the space of oriented lines $S^{2}$ in $\mathbb{R P}^{2}$ obtained by expressing the holonomy around each line in a fixed covariantly constant trivialisation of the bundle over $l_{\infty}$. Then if $A$ is sufficiently small, then $h$ determines $A$ uniquely up to gauge transformations.

The key ingredients of the proof of this theorem are contained in the Birkhoff factorization of equation (10), the definition of $H$ in equation (9) and the reconstruction of the connection from $H$ when $E$ is trivial of Theorem 2.

We note that the theorem in Novikov (2002) is actually expressed in terms of connections on $\mathbb{R}^{2}$ with suitable fall-of conditions, but they are easily seen to be slightly weaker than the statement above. This applies to our situation by considering $W_{\eta}$ to be a double cover of a projective plane $\mathbb{R P}^{2}$ using the joint antipodal map on $S^{2} \times S^{2}$. Under this map, great circles map to lines and $\partial W_{\eta}^{\prime-}$ can be taken to be a double cover of the 'line at $\infty$ ', $l_{\infty} ; W_{\eta}^{\prime-}-\partial W_{\eta}^{\prime-}$ then maps $1: 1$ to $\mathbb{R P}^{2}-l_{\infty}$.

It remains to prove that the connection that $H$ gives rise to on $\mathscr{I}^{-}$has holonomy given by $h$. This in fact is a special case of a more general proposition which is informative in its own right:

Proposition 5.1 Consider an ASDYM field on $\widetilde{\mathbb{M}}$ for which the twistor data has trivial vector bundle $E$ and is determined by a Hermitian metric $H$ on $\left.E\right|_{\mathbb{P T}_{\mathbb{R}}}$. $A$ pair $Z_{0}, Z$ of $\alpha$-planes in $\widetilde{\mathbb{M}}$ intersect in two antipodal points $x^{ \pm}$corresponding to the two orientations of the line $L$ in $\mathbb{P}_{\mathbb{R}}$ joining $Z_{0}$ and $Z$. Let $l$ be a loop in $Z_{0} \cup Z$ going from $x^{-}$to $x^{+}$in $Z_{0}$ and returning in $Z$. In a fixed covariantly constant frame on $Z_{0}, h_{l}$ is related to $\left.H\right|_{L}$ by

$$
h_{l}=g\left(x^{+}, Z\right) g\left(x^{-}, Z\right)^{-1},
$$

where $g\left(x^{ \pm}, Z\right)$ are the solutions to the Birkhoff factorizations

$$
H=g^{*}\left(x^{ \pm}, Z\right) g\left(x^{ \pm}, Z\right)
$$

such that $g\left(x^{ \pm}, Z\right)$ are respectively holmorphic on the two distinct holomorphic discs $D_{x^{ \pm}} \subset \mathbb{P} \mathbb{T}$ with boundary $L$ whose union is the complex projective line $\mathbb{C} L$ obtained by complexifying $L$.

Note that the holonomy $h_{l}$ of the ASDYM connection depends only on the topology of $l$ because the connection is flat on $\alpha$-planes.

Proof: In the construction of $(E, H)$ on $\mathbb{P T}$ from an ASDYM field on $\widetilde{\mathbb{M}}$, a frame of $E$ at a point $Z \in \mathbb{P T}_{\mathbb{R}}$ has the interpretation as a covariantly constant frame for the Yang-Mills bundle over the corresponding $\alpha$-plane $Z^{\prime}$ in $\widetilde{\mathbb{M}}$. On the other hand, in the factorization problem $H=g^{*}(x, Z) g(x, Z)$ where $g$ is defined holomorphically for $Z \in D_{x}, g$ has the interpretation as a map from a frame of $E_{Z}$ (i.e., covariantly constant on $\alpha_{Z}$ to a unitary frame of $E_{x}^{\prime}$. Putting these facts together, we see that, if we normalize $H\left(Z_{0}\right)=g\left(x^{ \pm}, Z_{0}\right)=I_{r}$, in a given unitary frame for $E_{Z_{0}}$, then $h_{l}$ as given in equation (12) is the product of the map from a covariantly constant frame along $Z_{0}$ to a covariantly constant frame along $Z$ at $x^{-}$with the map from the covariantly constant frame along $Z$ at $x^{+}$to that on $Z_{0}$ at $x^{+}$and is hence the holonomy as required. 
Corollary 5.1 If $H$ is defined according to equations (9) and (10), then the connection that $H$ gives rise to on $\mathscr{I}^{-}$has holonomy, as in definition [5.1 equal to the holonomy from which it was obtained.

Proof: We wish to calculate the holonomies $h(\widetilde{Z})$ of the Yang-Mills connection around the loops in $W_{\eta}^{\prime-}$ obtained by joining $\widetilde{Z}^{\prime}$ to one of the components of $\partial W_{\eta}^{\prime-}$. These should be evaluated in a frame that is covariantly constant around $\partial W_{\eta}^{\prime-}$. This is a special case of the above proposition as can be seen by taking $Z_{0}=Z_{\sigma_{\eta}}$ and $Z \in W_{\eta}$ so that $g\left(x^{ \pm}, Z\right)$ can be identified with $g^{ \pm}$of equations (109) and (11). Thus, given $H$, equations (11) and (9) can be considered to be the Birkhoff factorizations that give rise to a solution whose holonomy $h\left(z_{i}, \eta\right)$ is determined by (10) and hence gives the original $h\left(z_{i}, \eta\right)$ as required.

\subsection{Summary}

The scattering map from initial data $A_{-}$on $\mathscr{I}^{-}$to final data $A^{+}$on $\mathscr{I}^{+}$can be constructed by first constructing a family of parallel propagators $h(\widetilde{Z}): \widetilde{\mathbb{P T}}_{\mathbb{R}} \rightarrow$ $\mathrm{U}(n)$ along the intersections of the $\alpha$-planes with $\mathscr{I}^{-} ; h$ is defined on the double cover of twistor space. This can be used to find a hermitian metric $H$ on the restriction of a trivial bundle $E$ to $\mathbb{P T}_{\mathbb{R}}$ via the Birkhoff factorization problems (10, 9). Finally, $H$ can be used to recover an ASDYM field on $\widetilde{\mathbb{M}}$ and in particular final data $A^{+}$on $\mathscr{I}^{+}$via the proof of theorem [2 each of these maps is $1: 1$ and onto assuming small data.

\subsection{The nontriviality of the scattering}

We note that if we take theorem (15) as giving an equivalence between connections $A^{-}$ on $\mathscr{I}^{-}$and holonomy data $h(\widetilde{Z})$ on $\widetilde{\mathbb{P T}}_{\mathbb{R}}$, then the final data $A^{+}$is simply encoded in the holonomy data $h^{-1}(\widetilde{Z})$. This follows directly because the total holonomy around a curve in an $\alpha$-plane must be the identity as the curvature of an ASDYM connection is zero on $\alpha$-planes. In these terms, the scattering might seem rather trivial. In particular for $\mathrm{U}(1)$ connections we can see directly that there exists a gauge in which the scattering map is just reversal of sign: $A^{+}=-\tilde{\sigma}^{*} A^{-}$where $\tilde{\sigma}$ is the antipodal map. However, for non-abelian connections, there is no such simple formula relating $A^{+}$to $A^{-}$and the scattering at the level of the connection will be quite non-trivial. An appropriate geometrical analogue to consider here is the case of a Zoll surface which is a topological 2-sphere with a metric whose geodesics are all closed. Although Zoll perturbations of the round metric correspond to variations of the conformal factor that are odd under the antipodal map, finite Zoll perturbations are highly nontrivial and can only be completely characterised in the axisymmetric case, see for example LeBrun \& Mason (2002).

It is worth noting furthermore that theorem (5) is proved using the combination of Birkhoff factorizations above and these are non-trivially different for antipodal points $x^{+} \in \mathscr{I}^{+}$and $x^{-} \in \mathscr{I}^{-}$. The twistor data giving rise to the fields at $x^{ \pm}$ is simply $H$ restricted to the corresponding line $L_{x^{ \pm}}$which is the same line with the \pm determining opposite orientations. The discs $D_{x^{ \pm}}$meet at $L_{x^{ \pm}}$and make up a complex line. To construct the ASDYM field at $x^{ \pm}$, we solve the Birkhoff 
factorization problems

$$
H=g^{ \pm} g^{* \pm}
$$

where $g^{ \pm}$extend holomorphically over $D_{x^{ \pm}}$respectively. Since $D_{x^{ \pm}}$are complex conjugate discs in $\mathbb{P T}, g^{* \pm}$ extend holomorphically over $D_{x^{\mp}}$ respectively. Thus the two factorization problems in equation (14) are in opposite orders and will have non-trivially distinct solutions in the non-abelian case.

\section{$6 \quad$ Further developments}

There are a number of questions that remain.

One problem is to find interesting examples of the different types of ASDYM fields in split signature. We have constructed here solutions with $c_{1} \neq 0$ but $c_{2}=$ $c_{3}=0$, and in the appendix we have an example with $c_{2}=2, c_{1}=c_{3}=0$, but it would be interesting to have also an example with non-trivial $c_{3}$ or $\alpha$-invariant, but $c_{1}=c_{2}=0$. We also have not produced non-abelian examples with trivial $E$, but non-trivial $H$-such a solution would explicitly demonstrate the non-triviality of the scattering.

Perhaps the most interesting questions relate to the relationship between the constructions above and twistor-string theory. In particular, the version of twistorstring theory due to Berkovits, see for example Berkovits \& Witten (2004), focuses on curves in $\mathbb{P T}$ with boundary on $\mathbb{P T}_{\mathbb{R}}$. It would be intriguing to know how the constructions of this paper sit as an ASD sector inside those of Berkowitz which apply to the full Yang-Mills equations.

\section{A Proofs for theorem 2}

We first prove

Lemma (3.1): The given definition of $E \rightarrow \mathbb{P T}_{\mathbb{R}}$ is a smooth extension of $E$ from $\mathbb{P T}-\mathbb{P T}_{\mathbb{R}}$ such that the d-bar operator $\bar{\partial}_{E}$ extends smoothly over $\mathbb{P} \mathbb{T}_{\mathbb{R}}$.

Proof: Choose a small neighbourhood $U$ of $Z \in \mathbb{P T}_{\mathbb{R}}$ and a smooth frame $f$ of $E$ over $U_{\mathbb{R}}=U \cap \mathbb{P T}_{\mathbb{R}}$. This determines a smooth frame $q^{*} f$ of $E^{\prime}$ over $q^{-1}\left(U_{\mathbb{R}}\right)$ that is covariantly constant along the foliation of $q^{-1}\left(U_{\mathbb{R}}\right)$ by horizontal lifts of $\alpha$-planes. We extend this frame smoothly over $q^{-1} U$ as follows:

- We first construct a formal power series on $q^{-1}\left(U_{\mathbb{R}}\right)$ in a direction transverse to $q^{-1}\left(U_{\mathbb{R}}\right)$ in $q^{-1} U$ by requiring that the formal power series be holomorphic to all orders on the fibres of $p$ in $q^{-1} U \subset \mathscr{F}$. Each fibre of $p$ can be expressed as an upper-half plane $H$ on which $f$ is defined on some interval on the real axis. If $z=x+i y$ is a holomorphic coordinate on $H$ (say $z=\pi_{1^{\prime}} / \pi_{0^{\prime}}$ in some affine coordinate system as above), then the condition $\partial^{k} f / \partial \bar{z}^{k}=0$ for all $k$ determines $\partial^{k} f / \partial y^{k}$ uniquely for all $k$ inductively in terms of $\partial^{l} f / \partial x^{l}$ for $l \leq k$. Thus the formal power series is defined uniquely and hence globally.

- Using Borel's lemma, we can take a smooth extension of $f$ to $q^{-1} U$ whose power series on $q^{-1}\left(U_{\mathbb{R}}\right)$ is the given formal power series, and this can be made global by use of a partition of unity. 
In the frame thus constructed, we have that the d-bar operator on twistor space vanishes to all orders at $\mathbb{P} \mathbb{T}_{\mathbb{R}}$, and hence extends smoothly over $\mathbb{P} \mathbb{T}_{\mathbb{R}}$. This can be seen as follows.

The d-bar operator is given by

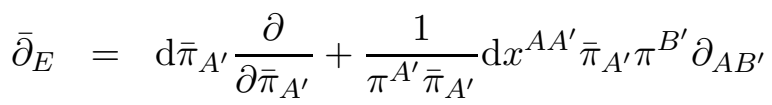

$$
\begin{aligned}
& +f^{-1}\left(\mathrm{~d} \bar{\pi}_{A^{\prime}} \frac{\partial}{\partial \bar{\pi}_{A^{\prime}}}+\frac{1}{\pi^{A^{\prime}} \bar{\pi}_{A^{\prime}}} \mathrm{d} x^{A A^{\prime}} \bar{\pi}_{A^{\prime}} \pi^{B^{\prime}} \mathrm{D}_{A B^{\prime}}\right) f
\end{aligned}
$$

where $D_{A A^{\prime}}$ is the given Yang-Mills connection. We claim that the potentially singular latter term $f^{-1}\left(\mathrm{~d} \bar{\pi}_{A^{\prime}} \frac{\partial}{\partial \bar{\pi}_{A^{\prime}}}+\frac{1}{\pi^{A^{\prime} \bar{\pi}_{A^{\prime}}}} \mathrm{d} x^{A A^{\prime}} \bar{\pi}_{A^{\prime}} \pi^{B^{\prime}} \nabla_{A B^{\prime}}\right) f$ vanishes at $\pi^{A^{\prime}} \bar{\pi}_{A^{\prime}}=0$ to all orders by construction. Firstly, the fact that $\partial f / \partial \bar{\pi}_{A^{\prime}}$ vanishes to all orders at $\pi^{A^{\prime}} \bar{\pi}_{A^{\prime}}=0$ follows immediately from the construction.

By definition of $\left.E\right|_{\mathbb{P}_{\mathbb{R}}}, \pi^{A^{\prime}} D_{A A^{\prime}} f=0$ when $\pi_{A^{\prime}}$ is real. Introducing an affine coordinate $z=\left(\pi_{0^{\prime}}+i \pi_{1^{\prime}}\right) /\left(\pi_{0^{\prime}}-i \pi_{1^{\prime}}\right)$ on the fibres of $\mathscr{F}$ (so that $\mathscr{F} \mathbb{R}$ is given by $|z|=1)$ the vanishing of $\partial f / \partial \bar{\pi}_{A^{\prime}}$ to all orders is equivalent to $\partial^{k} f / \partial \bar{z}^{k}$ for all $k$. Furthermore

$$
(\partial / \partial \bar{z})^{k}\left(\pi^{A^{\prime}} D_{A A^{\prime}} f\right)=\pi^{A^{\prime}} D_{A A^{\prime}}\left((\partial / \partial \bar{z})^{k} f\right)=0
$$

so that, by uniqueness of the extension, $\pi^{A^{\prime}} D_{A A^{\prime}} f=0$ to all orders on $q^{-1} U_{\mathbb{R}}$. Hence the latter, potentially singular terms, vanish to all orders at $U_{\mathbb{R}}$ and hence extend smoothly over $U_{\mathbb{R}}$ as desired.

\section{B Kahler structure and explicit solutions}

We develop a formalism for the correspondence in the case that a complex structure on $\widetilde{\mathbb{M}}$ is chosen. This reduces the symmetry group of twistor space from $\operatorname{PSL}(4, \mathbb{R})$ to PSO(4). The affine coordinates of $\$ 2.3$ respect the choice of a 'Lorentz' $\mathrm{SO}(2,2)$ subgroup of the space-time conformal group $\operatorname{PSO}(3,3)$, but here we will focus instead on a formalism that is invariant under the $\mathrm{SO}(3) \times \mathrm{SO}(3)$ subgroup of rigid rotations of each of the $S^{2}$ factors. This formalism is particularly well adapted to giving explicit descriptions of certain exact solutions which are described after the next subsection.

\section{B.1 The pseudo-kahler correspondence}

The space $\widetilde{\mathbb{M}}$ admits complex structures for which the metric (11) is scalar-flat pseudo-kahler. The complex structure realizes $\widetilde{\mathbb{M}}$ as the complex manifold $\mathbb{C P}^{1} \times \mathbb{C P}^{1}$ and via the representation of $\mathbb{P} \mathbb{T}-\mathbb{P T}_{\mathbb{R}}$ as the bundle of metric and orientation compatible complex structures over $\widetilde{\mathbb{M}}$, naturally embeds $\widetilde{\mathbb{M}}$ into $\mathbb{P T}$ as a quadric that misses $\mathbb{P T}_{\mathbb{R}}$.

On choosing a positive definite quadratic form $Q$ on $\mathbb{T}_{\mathbb{R}}$, we develop an $\mathrm{SO}(4)$ invariant formalism. Let $Z^{\alpha \dot{\alpha}}$ be coordinates on $\mathbb{T}$ where $\alpha=0,1$ and $\dot{\alpha}=\dot{0}, \dot{1}$ are $\mathrm{SU}(2)$ spinor indices for $\mathrm{SO}(4)=\mathrm{SU}(2) \times \mathrm{SU}(2) / \mathbb{Z}_{2}$ (we use different indices to avoid confusion with the $\mathrm{SL}(2, \mathbb{R})$ spinor indices used above for $\mathrm{SO}(2,2))$. Recall that for $\mathrm{SU}(2)$ spinors we raise and lower indices with the alternating spinors 
$\varepsilon_{\alpha \beta}=-\varepsilon_{\beta \alpha}, \varepsilon_{\dot{\alpha} \dot{\beta}}=-\varepsilon_{\dot{\beta} \dot{\alpha}}, \varepsilon_{01}=\varepsilon_{\dot{0} \dot{1}}=1$. We also have the quaternionic complex conjugation $x^{\alpha} \rightarrow \hat{x}^{\alpha}=\left(-\bar{x}^{1}, \bar{x}^{0}\right)$ so that $\mathbb{T}_{\mathbb{R}}$ consists of those $Z^{\alpha \dot{\alpha}}$ such that $\hat{Z}^{\alpha \dot{\alpha}}=Z^{\alpha \dot{\alpha}}$.

Points of $\widetilde{\mathbb{M}}$ can be identified with points of the quadric $Q \subset \mathbb{P T}$ (using an obvious abuse of notation)

$$
Q=\left\{Z \in \mathbb{P} \mathbb{T} \mid Q:=Z^{\alpha \dot{\alpha}} Z_{\alpha \dot{\alpha}}=0\right\} .
$$

This gives $Z^{\alpha \dot{\alpha}}=x^{\alpha} y^{\dot{\alpha}}$ on $Q$ and each of $x^{\alpha}$ and $y^{\dot{\alpha}}$ can be thought of as homogeneous coordinates on the $\mathbb{C P}^{1}$ factors of $\widetilde{\mathbb{M}}=\mathbb{C P}^{1} \times \mathbb{C P}^{1}$ as a complex manifold.

We take $\left(x^{\alpha}, y^{\dot{\alpha}}\right)$ to be homogeneous coordinates on $\widetilde{\mathbb{M}}$ normalized so that $x^{\alpha} \hat{x}_{\alpha}=y^{\alpha} \hat{y}_{\alpha}=1$ related to the previous affine coordinates by $w_{1}=x^{1} / x^{0}$ and $w_{2}=y^{\dot{1}} / y^{\dot{0}}$. Homogeneous functions $f\left(x^{\alpha}, y^{\alpha}\right)$ will be said to have weight $(p, q)$ if $f\left(\mathrm{e}^{i \theta} x^{\alpha}, \mathrm{e}^{i \phi} y^{\alpha}\right)=\mathrm{e}^{(p i \theta+q i \phi)} f\left(x^{\alpha}, y^{\alpha}\right)$. Such homogeneous functions can also be taken to represent sections of the tensor product of $p$ copies of the spin bundle on the first $S^{2}$ factor with $q$ copies of the spin bundle on the second factor.

We can introduce homogenous coordinates $\left(x^{\alpha}, y^{\alpha}, \lambda_{A}\right)$ on $\mathscr{F}$ where $\lambda=\lambda_{1} / \lambda_{0}$, $|\lambda| \leq 1$ is a coordinate on the unit disc $D_{x}$, and $\lambda_{0}$ has weight $(-1,-1), \lambda_{1}$ has weight $(1,1)$ so that $\lambda$ has weight $(2,2)$ and the incidence relation is

$$
Z^{\alpha \dot{\alpha}}=\lambda_{0} x^{\alpha} y^{\dot{\alpha}}+\lambda_{1} \hat{x}^{\alpha} \hat{y}^{\dot{\alpha}}
$$

This can be expressed implicitly as

$$
Z_{\alpha \dot{\alpha}} x^{\alpha} \hat{y}^{\dot{\alpha}}=Z_{\alpha \dot{\alpha}} \hat{x}^{\alpha} y^{\dot{\alpha}}=0
$$

which can be seen to reduce to one complex equation when $Z^{\alpha \dot{\alpha}}$ is real yielding the formula $x^{\alpha} \propto Z_{\dot{\alpha}}^{\alpha} \hat{y}^{\dot{\alpha}}$ for real $\alpha$-planes, i.e., a real $\alpha$-plane is the graph of an orientation reversing isometry from the second to the first factor.

In these coordinates the Lax pair can be expressed in terms of the holomorphic and antiholomorphic exterior derivatives $\mathrm{\partial}_{x}, \overline{\widehat{\partial}}_{x}$ and $\widetilde{\partial}_{y}, \overline{\mathrm{\partial}}_{y}$ on the $S^{2}$ factors with homogeneous coordinates $x^{\alpha}, y^{\dot{\alpha}}$ respectively. Rather than taking these as form valued operators we can instead can take them to have weights $(-2,0),(2,0),(0,-2)$ and $(0,2)$ respectively. We have

$$
L_{0}=\lambda ð_{x}-\overline{\widetilde{\partial}}_{y}, \quad L_{1}=\lambda \varlimsup_{y}-\overline{\check{\partial}}_{x}
$$

$L_{0}$ and $L_{1}$ define the complex structure on the complement of $\partial \mathscr{F}$ and are tangent to the fibration $\partial \mathscr{F} \rightarrow \mathbb{P} \mathbb{T}_{\mathbb{R}}$.

\section{B.2 ADHM description of instantons with $c_{2}=2$}

Since the split-signature instantons correspond to stable bundles on $\mathbb{P T}$ they can be constructed using an adaptation of the ADHM construction to split signature. The ADHM construction expresses a rank $n$ bundle $E \rightarrow \mathbb{P T}$ with $c_{2}=k$ as the cohomology of the sequence

$$
V \stackrel{K \cdot Z}{\longrightarrow} W \stackrel{K^{*} \cdot Z}{\longrightarrow} \bar{V}^{*}
$$

Here $V, W$ are complex vector space of dimension $k$ and $2 k+n$ respectively, $W$ has a pseudo-Hermitian metric $h$ (which is used to deine the $*$ operation below) and 
$K: V \otimes \mathbb{T} \rightarrow W$ are linear maps such that $K^{*} \cdot Z \circ K \cdot Z=0$ for all $Z$. All bundles corresponding to instantons arise in this way. Given the ADHM data of the matrices $K$, the corresponding solution on space-time can be written down explicitly, Atiyah (1979) and will be non-singular if certain non-degeneracy conditions are satisfied.

Here we work through the first non-trivial case of $k=n=2$. In this case we can choose our frames of $\mathbb{T}, V$ and $W$ so that coordinates on these spaces have the index structure $Z^{\alpha \dot{\alpha}}$ as above, $v^{\alpha}$ and $w^{\alpha_{1} \alpha_{2} \dot{\alpha}}=w^{\left(\alpha_{1} \alpha_{2}\right) \dot{\alpha}}$ respectively. In this frame the map $K$ is $K_{\alpha \beta \dot{\beta}}^{\gamma_{1} \gamma_{2} \dot{\gamma}}=\delta_{\alpha}^{\left(\gamma_{1}\right.} \delta_{\beta}^{\left.\gamma_{2}\right)} \delta_{\dot{\beta}}^{\dot{\gamma}}$ and the hermitian metric on $W$ is

$$
h(w, w)=w^{\alpha_{1} \alpha_{2} \dot{\alpha}} \hat{w}^{\beta_{1} \beta_{2} \dot{\beta}} h_{\alpha_{1} \alpha_{2} \beta_{1} \beta_{2}} i \varepsilon_{\dot{\alpha} \dot{\beta}},
$$

where $h_{\alpha \beta \gamma \delta}=h_{(\alpha \beta \gamma \delta)}=\hat{h}_{\alpha \beta \gamma \delta}$ and $v^{\alpha} \rightarrow \hat{v}^{\alpha}$ is the standard quaternionic $\mathrm{SU}(2)$ conjugation. With this, the ADHM equations $K^{*} \cdot Z \circ K \cdot Z=0$ are satisfied.

In order to write down the corresponding solution on space-time, it is convenient to use the representation of space-time above as the quadric $Q$. A point $x \in \mathbb{M}$ is represented by the pair of spinors $\left(x^{\alpha}, y^{\dot{\alpha}}\right)$ which correspond to the line joining $Z_{1}^{\alpha \dot{\alpha}}=x^{\alpha} y^{\dot{\alpha}}$ and $Z_{2}^{\alpha \dot{\alpha}}=\hat{x}^{\alpha} \hat{y}^{\dot{\alpha}}$ in $\mathbb{P T}$. The fibre $E_{x}^{\prime}$ of $E^{\prime} \rightarrow \mathbb{M}$ at $x$ can be represented as the subspace of $W$ in the kernel of $K^{*} \cdot Z_{1}$ and $K^{*} \cdot Z_{2}$. The projector $P_{x}: W \rightarrow W$ onto the subspace $E_{x}^{\prime}$ can be constructed as

$$
P_{x}=I-K \cdot Z_{1} \Delta(x)^{-1} K^{*} \cdot Z_{2}+K \cdot Z_{2} \Delta(x)^{-1} K^{*} \cdot Z_{1}
$$

where

$$
\Delta(x):=K^{*} \cdot Z_{2} \circ K \cdot Z_{1}=-K^{*} \cdot Z_{1} \circ K \cdot Z_{2}
$$

and the latter identity follows from the ADHM equation. A smooth (but not holomorphic) unitary frame for $E_{x}^{\prime} \subset W$ is given explicitly by

$$
U=\frac{1}{\sqrt{h^{-1 \alpha_{1} \alpha_{2} \beta_{1} \beta_{2}} x_{\alpha_{1}} x_{\alpha_{2}} \hat{x}_{\beta_{1}} \hat{x}_{\beta_{2}}}}\left\{y^{\dot{\alpha}} h^{-1 \alpha_{1} \alpha_{2} \beta_{1} \beta_{2}} \hat{x}_{\beta_{1}} \hat{x}_{\beta_{2}}, \hat{y}^{\dot{\alpha}} h^{-1 \alpha_{1} \alpha_{2} \beta_{1} \beta_{2}} x_{\beta_{1}} x_{\beta_{2}}\right\}
$$

The connection is given by projecting infinitesimally $E_{x}$ to $E_{x+\delta x}$ inside $W$, thus

$$
\nabla U=P_{x} \mathrm{~d} U
$$

which can now be calculated explicitly by the reader with the energy and inclination.

We note that although a formalism based on the double cover $\widetilde{\mathbb{M}}$ of $\mathbb{M}$ has been used here, the formula for $P_{x}$ is invariant under the antipodal map and the solution descends to $\mathbb{M}$.

The construction breaks down when $\Delta(x)=x^{\gamma} \hat{x}^{\delta} h_{\gamma \delta \alpha \beta}$ is singular. The determinant of $\Delta(x)$ is $d=x^{\gamma} \hat{x}^{\delta} h_{\gamma \delta \alpha \beta} x^{\epsilon} \hat{x}^{\phi} h_{\epsilon \phi}^{\alpha \beta}$ and so $\Delta$ is non-degenerate for all $x$ if $h$ is non-degenerate when regarded as a symmetric trace-free $3 \times 3$ matrix over spinors $V^{\alpha \beta}=v^{(\alpha \beta)}$. (It is worth noting that the locus $d=0$ in the complex is the same as that defining the jumping lines as described in 4.3 given by $S(\mathbf{x}, \mathbf{x})=\mathbf{x} \cdot \mathbf{x}$ where $S-1$ is identified with a constant multiple of $h_{\alpha_{1} \alpha_{2} \gamma_{1} \gamma_{2}} h_{\beta_{1} \beta_{2}}^{\gamma_{1} \gamma_{2}}$ where the symmetric pairs of spinor indices $\alpha_{1} \alpha_{2}$ are identified with 3-vector indices in the standard way; it can be checked that the condition that $S$ arises from a traceless spinor in this way is equivalent to the Poncelet condition $\operatorname{tr} S^{2}=3 / 2$.) 


\section{B.3 Yang's J-matrix formulation}

On a (pseudo-)kahler 4-manifold, the ASDYM equations can be recast as the condition that the bundle with unitary connection $\left(E^{\prime}, D\right)$ be compatible with the complex structure and satisfy in addition the condition that $\omega \wedge F=0$ where $\omega$ is the Kahler 2-form and $F$ is the curvature of the connection. Given a holomorphic vector bundle, Chern's theorem states that unitary connections compatible with the given complex structure are in 1:1 correspondence with hermitian metrics $J$ on $E^{\prime}$. In a local holomorphic frame, the connection on the bundle is obtained by differentiation of $J$ and the ASDYM equation is given by $\omega \wedge \bar{\partial}\left(J^{-1} \partial J\right)=0$. In the physics literature, $J$ has become known as Yang's J-matrix.

In the simplest situation, $E^{\prime}$ will be trivial as a holomorphic vector bundle over $\widetilde{\mathbb{M}}=\mathbb{C P}^{1} \times \mathbb{C P}^{1}$ and so the holomorphic frame will be defined globally up to constant $\mathrm{GL}(n, \mathbb{C})$ transformations. Thus so will $J$.

If $E$ and $E^{\prime}$ are both trivial, then $J$ and $H$ will be related by $J\left(x^{\alpha}, y^{\dot{\alpha}}\right)=$ $g\left(x^{\alpha} y^{\dot{\alpha}}\right)^{-1} g\left(x^{\alpha} y^{\dot{\alpha}}\right)^{*-1}$ where $g$ is as defined in equation (8).

\section{B.4 Explicit solutions from Ward ansatze}

We consider the ansatze of 4.2 and work throught the procedure to obtain the $J$-matrix of the bundle on $\widetilde{\mathbb{M}}$. We note that, as a holomorphic vector bundle over $Q, E^{\prime}$ is non-trivial since it is the restriction of $E=\mathcal{O}(1) \oplus \mathcal{O}(-1)$ to $Q$ from $\mathbb{P}$. On $Q, E^{\prime}=\left.E\right|_{Q}=\mathcal{O}(1,1) \oplus \mathcal{O}(-1,-1)$ where $\mathcal{O}(p, q)$ is defined to be the tensor product of the pullback of $\mathcal{O}(p)$ from the first $\mathbb{C P}^{1}$ factor (coordinatised by $x^{\alpha}$ ) with $\mathcal{O}(q)$ from the second one (coordinatised by $y^{\dot{\alpha}}$ ). Thus, the $J$ matrix can only be presented globally if its entries are understood to take values in the appropriate line bundles.

In order to make clear the holomorphic nature of these line bundles we do not in the following normalize $x^{\alpha} \hat{x}_{\alpha}=1$ etc.. We also use homogeneous coordinates $\left(\lambda_{0}, \lambda_{1}\right)$ on the discs $D_{x}$ so that $\lambda=\lambda_{1} / \lambda_{0}$ and the incidence relation becomes

$$
Z^{\alpha \dot{\alpha}}=\lambda_{0} x^{\alpha} y^{\dot{\alpha}}+\lambda_{1} \hat{x}^{\alpha} \hat{y}^{\dot{\alpha}}
$$

Note that when $Z^{\alpha \dot{\alpha}}$ is real, $\lambda \bar{\lambda}=1$, i.e., $\bar{\lambda}=1 / \lambda$. In terms of homogeneous coordinates we have $\left(\lambda_{0}, \lambda_{1}\right)=\left(\bar{\lambda}_{1}, \bar{\lambda}_{0}\right)$.

We consider the adaptation of the Ward ansatze given in 4.2 in the case that $k=1$ and $Q$ is the quadric as above. We will take $f$ to be an arbitrary smooth function on $\mathbb{P T}_{\mathbb{R}}$ corresponding to an arbitrary smooth solution $\phi$ to the ultrahyperbolic wave equation on $\mathbb{M}$ by means of the X-ray transform of the function $f / Q$ which in these coordinates becomes

$$
\phi(x)=\frac{1}{2 \pi i} \oint_{|\lambda|=1} f\left(x^{\alpha} y^{\dot{\alpha}}+\lambda \hat{x}^{\alpha} \hat{y}^{\dot{\alpha}}\right) \frac{\mathrm{d} \lambda}{\lambda} .
$$

An intermediate step in finding the solutions in the abelian case with patching function is $\mathrm{e}^{f}$ is to find a function $g(x, \lambda)$ holomorphic in $\lambda$ on each disc $D_{x}=\{|\lambda| \leq$ 1 ) such that $f=g+\bar{g}$ on $\partial D_{x}$. Such a $g$ can be obtained by the integral formula

$$
g(x, \lambda)=\frac{1}{2 \pi i} \oint_{\left|\lambda^{\prime}\right|=1} f\left(x^{\alpha} y^{\dot{\alpha}}+\lambda^{\prime} \hat{x}^{\alpha} \hat{y}^{\dot{\alpha}}\right) \frac{\mathrm{d} \lambda^{\prime}}{\left(\lambda^{\prime}-\lambda\right)} .
$$


Clearly $g$ is unique up to the addition of an imaginary constant which, in the formula above, has been chosen so that $g_{0}:=g(x, 0)=\phi(x)$.

The key step in finding the ASDYM field from the ansatze is to find the matrix functions $G(x, \lambda)$ holomorphic in $|\lambda| \leq 1$ that satisfy $G H G^{*}=I$ on $|\lambda|=1$ for the given

$$
H=\left(\begin{array}{cc}
2 Q^{-1} \cosh f & \mathrm{e}^{-f} \\
\mathrm{e}^{-f} & Q \mathrm{e}^{-f}
\end{array}\right)
$$

If we set

$$
G=\left(\begin{array}{ll}
a & b \\
c & d
\end{array}\right)
$$

we should take $a, c$ to be sections of $\mathcal{O}(1)$ and $b, d$ of $\mathcal{O}(-1)$ that are holomorphic over $D_{x}=\{|\lambda| \leq 1\}$ such that

$$
a \mathrm{e}^{f}=\bar{c}+Q \bar{d}, \quad \text { and } \quad(a+b Q) \mathrm{e}^{-f}=-\bar{c} .
$$

These equations can be solved directly by expressing $f$ in terms of $g$ as above so that

$$
(a+b Q) \mathrm{e}^{-g}=-\mathrm{e}^{\bar{g}} \bar{c}, \quad \text { and } \quad a \mathrm{e}^{g}=(\bar{c}+Q \bar{d}) \mathrm{e}^{-\bar{g}} .
$$

These expressions therefore determine sections of $\mathcal{O}(1)$ over $\partial D_{x}$ that extend over $D_{x}$ holomorphically and whose complex conjugates do also. By an application of an extension of Liouville's theorem, they must therefore be the restriction of global sections $\beta^{A} \lambda_{A}$ and $\alpha^{A} \lambda_{A}$ of $\mathcal{O}(1)$ where $\alpha^{A}$ and $\beta^{A}$ are independent of $\lambda^{A} .{ }^{10}$

This gives for $a, b, c$ and $d$

$$
a=\mathrm{e}^{-g} \alpha^{A} \lambda_{A}, b=\frac{\mathrm{e}^{g} \beta^{A} \lambda_{A}-\mathrm{e}^{-g} \alpha^{A} \lambda_{A}}{Q}, c=-\mathrm{e}^{-g} \bar{\beta}^{A} \lambda_{A}, d=\frac{\mathrm{e}^{g} \bar{\alpha}^{A} \lambda_{A}+\mathrm{e}^{-g} \bar{\beta}^{A} \lambda_{A}}{Q}
$$

However, we require that at $Q=0, b$ and $d$ are regular and this requires that the numerators of the fractions vanish there also. Using $g(x, 0)=\phi$, this gives the relations

$$
\beta^{0}=\mathrm{e}^{-2 \phi} \alpha^{0}, \quad \text { and } \quad \bar{\beta}^{1}=-\mathrm{e}^{2 \phi} \bar{\alpha}^{1} .
$$

These determine $\beta^{A}$ in terms of $\alpha^{A}$ and reduce the unit determinant condition to

$$
1=a d-b c=\left(1+\mathrm{e}^{-4 \phi}\right) \alpha^{0} \bar{\alpha}^{0}+\left(1+\mathrm{e}^{4 \phi}\right) \alpha^{1} \bar{\alpha}^{1} .
$$

The $J$-matrix is $J=G^{-1}(x, 0) G^{*-1}(x, 0)$ and calculation yields that at $\lambda=0$ :

$$
\begin{gathered}
a=\mathrm{e}^{-\phi} \alpha^{0} \lambda_{0}, \quad b=-\mathrm{e}^{-g} \frac{\alpha^{1}\left(1+\mathrm{e}^{4 \phi}\right)-2 \alpha^{0} g_{0}^{\prime}}{\lambda_{0}}, \\
c=\mathrm{e}^{\phi} \bar{\alpha}^{1} \lambda_{0}, \quad d=\mathrm{e}^{\phi} \frac{2 \bar{\alpha}^{1} g_{0}^{\prime}+\bar{\alpha}_{0}\left(1+\mathrm{e}^{-4 \phi}\right)}{\lambda_{0}}
\end{gathered}
$$

\footnotetext{
${ }^{10}$ The standard argument is that if both a function $g$ on $\partial D_{x}$ and its complex conjugate $\bar{g}$ extend holomorphically over $D_{x}$, then its real and imaginary parts extend holomorphically over $D_{x}$ also and must therefore be constant. Alternatively, a holomophic function $f$ on $D_{x}$ that is real on $\partial D_{x}$ is constant since it can be extended to a bounded holomorphic function $\overline{f(1 / \bar{\lambda})}$ on the complex plain by inversion and continuity at $\partial D_{x}$. Here, the complex conjugates simply extend holomorphically over $D_{x}$ as $\bar{\beta}^{A} \lambda_{A}$ and $\bar{\alpha}^{A} \lambda_{A}$ since $\lambda_{A}=\bar{\lambda}_{A}$ on $\partial D_{x}$ ).
} 
where $g_{0}^{\prime}=\mathrm{d} g /\left.\mathrm{d} \lambda\right|_{\lambda=0}$. This gives

$$
J=\left(\begin{array}{cc}
\frac{2\left(\cosh ^{2} 2 \phi+g_{0}^{\prime} \bar{g}_{0}^{\prime}\right)}{\lambda_{0}^{2} \cosh 2 \phi} & \frac{-g_{0}^{\prime}}{\cosh 2 \phi} \\
\frac{-\bar{g}_{0}^{\prime}}{\cosh 2 \phi} & \frac{\lambda_{0}^{2}}{2 \cosh 2 \phi}
\end{array}\right) .
$$

In this formula we note the appearance of $\lambda_{0}$ which is a coordinate up the fibre of $\mathcal{O}(-1,-1)$. A section $\left(s_{0}, s_{1}\right)$ of $\mathcal{O}(1,1) \oplus \mathcal{O}(-1,-1)$ is here being understood as being represented concretely by the homogeneous functions $\left(s_{0} / \lambda_{0}, s_{1} \lambda_{1}\right)$ and it is on expressions of this form that $J$ provides a hermitian metric.

We also note the appearance of the function $g_{0}^{\prime}(x)$. This from equation (16) can be expressed as

$$
g^{\prime}(x, 0)=\frac{1}{2 \pi i} \oint_{\left|\lambda^{\prime}\right|=1} f\left(x^{\alpha} y^{\dot{\alpha}}+\lambda^{\prime} \hat{x}^{\alpha} \hat{y}^{\dot{\alpha}}\right) \frac{\mathrm{d} \lambda^{\prime}}{\lambda^{2}} .
$$

This can be obtained from $\phi$ as follows. Recall that the Lax pair on the spin bundle can be represented in this context by $L_{0}=\lambda ð_{x}-\bar{\partial}_{y}$ and $L_{1}=\lambda ð_{y}-\bar{\partial}_{x}$. Then, $L_{0} f=L_{1} f=0$ so that differentiation of equations (1517) gives

$$
\overline{\mathrm{\partial}}_{x} g_{0}^{\prime}=\widetilde{\mathrm{\partial}}_{y} \phi, \quad \text { and } \overline{\mathrm{\partial}}_{y} g_{0}^{\prime}=\mathrm{\partial}_{x} \phi \text {. }
$$

Given $\phi$ satisfying the wave equation, these equations are integrable and can be solved for $g_{0}^{\prime}$ in terms of $\phi$.

As a final comment, we note that the wave equation on $\phi$ in this context is simply

$$
\Delta_{x} \phi=\Delta_{y} \phi
$$

where $\Delta_{x}$ and $\Delta_{y}$ are the round sphere Laplacians on the $x^{A}$ and $y^{A^{\prime}}$ spheres respectively. The equation therefore clearly has separable solutions given as the product of spherical harmonics $\phi=Y_{l m}\left(x^{\alpha}\right) Y_{l m^{\prime}}\left(y^{\dot{\alpha}}\right)$ on each factor.

\section{References}

Ablowitz, M. and Clarkson, P.A. (1991) Solitons, nonlinear evolution equations and inverse scattering, LMS lecture notes series 149, CUP.

Atiyah, M.F. (1979) Geometry of Yang-Mills fields, Accademia Nazionale dei Lincei Scuola Normale Superiore, Lezione Fermiane, Pisa.

Atiyah, M.F., Hitchin, N., and Singer, I. (1978) Self-duality in four-dimensional Riemannian geometry, Proc. Roy. Soc. Lond., A 362, 425-61.

Atiyah, M.F., \& Rees, E.G. (1976) Vector bundles on projective 3-space, Inventiones Mathematicae, 35, 131-53.

Bailey, T.N. (1985) Twistors and fields with sources on worldlines, Proc. Roy. Soc. Lond., A397, 143-55.

Bailey, T.N., Eastwood, M.G., Gover, R., and Mason, L.J. (1999) The Funk transform as a Penrose transform, Math. Proc. Camb. Phil. Soc., 125, no. 1. p67-81.

Bailey, T.N., Eastwood, M.G., Gover, R., and Mason, L.J. (2003) Complex analysis and the Funk transform, J. Korean Math Soc., bf 40, no. 4, 577-593. 
Bailey, T.N., Eastwood (2001) Twistor results for integral transforms, in Radon transforms and tomography (South Hadley, MA, 2000), Contemp. Math. 278, p77-86.

Belavin, A.A., and Zakharov, V.E. (1978) Yang-Mills equations as inverse scattering problem, Phys. Lett., 73B, 53-7.

Berkovits, N. and Witten, E. (2004) Conformal gravity in twistor-string theory, hep-th/0406051, see also hep-th/0402045 and hep-th/0403187.

Chalmers, G. and Siegel, W. (1996) Self-dual sector of QCD amplitudes, Phys. Rev. D, 54, no. 12, 7628-33.

Dunajski, M., (2002) Anti-self-dual 4-manifolds with a parallel real spinor. Proc. Roy. Soc. Lond. A, 458, no. 2021, p1205-22.

Faddeev, L.D. and Takhtajan, L.A. (1987) Hamiltonian methods in the theory of solitons, Springer-Verlag, Berlin-Heidelberg-New York.

John, F. (1938) The ultrahyperbolic differential equation with four independent variables, Duke Math. J., 4, 300-322, reprinted in 75 years of the Radon transform (Vienna 1992), Conf. Proc. Lecture Notes Math. Phys., IV, p301-323, International Press.

Gohberg, I.C., and Krein, M.G. (1958) Systems of integral equations on the half-line with kernels depending on the differences of the arguments. Uspekhi Mat. Nauk, 13, 3-72. (Russian)

Guillemin, V. \& Sternberg, S. (1986) An ultra-hyperbolic analogue of the RobinsonKerr theorem. Lett. Math. Phys., no. 1, 1-6.

Kotecha, V., Ward, R.S. (2001) Integrable Yang-Mills-Higgs equations in threedimensional de Sitter space-time, J. Math. Phys., 42, No. 3, 1018-1025.

LeBrun and Mason (2002) Zoll manifolds and complex surfaces, J. Diff. Geom., 61, 453-535.

LeBrun and Mason (2005) Nonlinear Gravitons, Null Geodesics and Holomorphic Discs, math.DG/0504582.

Lerner, D.E. (1992) The linear system for self-dual gauge fields in a space-time of signature 0, J. Geom. Phys, 8, 211-9.

Manakov, S.V., \& Zakharov, V.E. (1981) Three dimensional model of relativisticinvariant field theory, integrable by the inverse scattering transform, Lett. Math. Phys., 5, 247-253.

Hughston, L.P., \& Mason, L.J. (1990) Further Advances in Twistor Theory, Vol. I: The Penrose tranform and its applications, Pitman Res. notes in Math. Ser., 231, Longman.

Mason, L.J. (1995) Global solutions of the self-duality equations in split signature, in Further Advances In twistor Theory, Vol II: Integrable Systems, conformal geometry and gravitation, eds L.J.Mason, L.P.Hughston \& P.Kobak, Pitman research Notes in Maths Series, 232, Longman Scientific and Technical / Wiley.

Mason, L.J. \& Woodhouse, N.M.J. (1996) Integrability, self-duality and twistor theory, OUP.

Novikov, R. (2002) On determination of a gauge field on $\mathbb{R}^{d}$ from its non-abelian Radon transform along oriented straight lines, J.Inst. Math. Jussieu, 1, 4, 559-629. 
Penrose, R. (1963 reprinted 1980) Null hypersurface initial data for classical fields of arbitrary spin and for general relativity, in Aerospace Research Laboratories Report 63-56 (P.G.Bergmann) reprinted (1980) in Gen. Rel. Grav., 12, 225-64.

Penrose, R. (1976) Nonlinear gravitons and curved twistor theory, Gen. Rel. Grav. 7, 31-52.

Penrose, R., \& Rindler, W. (1984 \& 1986) Spinors \& Space-times Vol. I 63 II, CUP. Rendall, A. (1992) The characteristic initial value problem for the Einstein equations, Nonlinear hyperbolic equations and field theory (Lake Como, 1990), p154-163, Pitman Res. Notes Maths Ser., 253.

Sparling, G.A.J. (1998) Inversion for the Radon line transform in higher dimensions, Phil. Trans. Roy. Soc. Ser. A, 356, no. 1749, p3041-3086.

Ward, R.S. (1977) On self-dual gauge fields, Phys. Lett., 61A, 81-2.

Ward, R.S., (1979) Massless fields from twistor functions, §2.4 in Advances in Twistor Theory, eds Hughston \& Ward, Pitman Research Notes in Math., 37, Pitman.

Ward, R.S. (1995) Nontrivial scattering of localized solitons in a (2+1)-dimensional integrable system. Phys. Lett. A, 208, no. 3, 203-8.

Ward, R.S. (1999) Two integrable systems related to hyperbolic monopoles, in Sir Michael Atiyah: A great mathematician of the twentieth century, Asian J.Math., 3, no. $1,325-332$.

Ward, R.S., \& Wells, R.O. (1990) Twistor geometry and field theory, CUP.

Witten, E. (2003) Perturbative gauge theory as a string theory in twistor space, hep-th/0312171.

Woodhouse, N.M.J. (1992) Contour integrals for the ultrahyperbolic wave equation, Proc. Roy. Soc. London, A438, 197-206. 